\title{
Einstein-Podolsky-Rosen-like separability indicators for two-mode Gaussian states
}

\author{
Paulina Marian 1 , 2* and Tudor A. Marian时 \\ ${ }^{1}$ Centre for Advanced Quantum Physics, Department of Physics, \\ University of Bucharest, R-077125 Măgurele, Romania and \\ ${ }^{2}$ Department of Physical Chemistry, University of Bucharest, \\ Boulevard Regina Elisabeta 4-12, R-030018 Bucharest, Romania
}

\begin{abstract}
We investigate the separability of the two-mode Gaussian states by using the variances of a pair of Einstein-Podolsky-Rosen (EPR)-like observables. Our starting point is inspired by the general necessary condition of separability introduced by Duan et al. [Phys. Rev. Lett. 84, 2722 (2000)]. We evaluate the minima of the normalized forms of both the product and sum of such variances, as well as that of a regularized sum. Making use of Simon's separability criterion, which is based on the condition of positivity of the partial transpose (PPT) of the density matrix [Phys. Rev. Lett. 84, 2726 (2000)], we prove that these minima are separability indicators in their own right. They appear to quantify the greatest amount of EPR-like correlations that can be created in a two-mode Gaussian state by means of local operations. Furthermore, we reconsider the EPR-like approach to the separability of two-mode Gaussian states which was developed by Duan et al. with no reference to the PPT condition. By optimizing the regularized form of their EPR-like uncertainty sum, we derive a separability indicator for any two-mode Gaussian state. We prove that the corresponding EPR-like condition of separability is manifestly equivalent to Simon's PPT one. The consistency of these two distinct approaches (EPR-like and PPT) affords a better understanding of the examined separability problem, whose explicit solution found long ago by Simon covers all situations of interest.

PACS numbers: 03.67.-a, 42.50.Dv, 03.65.Ud
\end{abstract}

\section{INTRODUCTION}

Detecting and measuring quantum entanglement represent one of the goals of quantum information science. In the last two decades, a large amount of work has been invested in writing efficient separability criteria for both discrete- and continuous-variable systems. Although bipartite entanglement seems to be the simplest to detect and measure, practically we are still forced to apply different criteria when discussing it for mixed states of composite systems.

As shown by Peres [1], a necessary condition of separability for an arbitrary two-party state is the requirement to have a non-negative partially transposed density matrix. In the case of discrete-variable systems, this requirement of positive partial transposition (PPT) is also a sufficient condition of separability only for states on $\mathbb{C}^{2} \otimes \mathbb{C}^{2}$ and $\mathbb{C}^{2} \otimes \mathbb{C}^{3}$ Hilbert spaces [2]. For bipartite states of continuous-variable systems, the PPT condition was first applied by Simon [3]. Specifically, Simon proved that preservation of the non-negativity of the density matrix under partial transposition is not only a necessary, but also a sufficient condition for the separability of twomode Gaussian states (TMGSs). Moreover, the partial transposition criterion could be expressed in an elegant symplectically invariant form valid for any TMGS [3]. To detect the continuous-variable entanglement for nonGaussian states, Shchukin and Vogel have derived an in-

\footnotetext{
* paulina.marian@g.unibuc.ro

$\dagger$ tudor.marian@g.unibuc.ro
}

finite series of inequalities for the moments of the state required by the PPT condition [4]. Similar inequalities were obtained in Refs. [5, 6].

A somewhat parallel method to get general inseparability criteria for two-mode states originates in a practical procedure proposed by Reid for demonstrating the Einstein-Podolsky-Rosen (EPR) paradox [7] in a nondegenerate parametric amplifier [8]. This was done by using two non-local observables linearly built with the canonical quadrature operators of the modes, $\hat{q}_{j}, \hat{p}_{j},(j=$ $1,2)[8,9]:$

$$
\hat{Q}(\lambda):=\hat{q}_{1}-\lambda \hat{q}_{2}, \quad \hat{P}(\mu):=\hat{p}_{1}+\mu \hat{p}_{2},
$$

where $\lambda$ and $\mu$ are adjustable positive parameters. As a consequence of their commutation relation,

$$
[\hat{Q}(\lambda), \hat{P}(\mu)]=i(1-\lambda \mu) \hat{I},
$$

we get the weak (Heisenberg) form of the uncertainty principle,

$$
\Delta Q(\lambda) \Delta P(\mu) \geqq \frac{1}{2}|1-\lambda \mu|,
$$

which has to be fulfilled by any quantum state. In Eq. (1.3) and in the sequel as well, $(\Delta A)_{\hat{\rho}}$ denotes the standard deviation of the observable $\hat{A}$ in the state $\hat{\rho}$, which is the square root of the variance

$$
\left[(\Delta A)_{\hat{\rho}}\right]^{2}:=\left\langle\left(\hat{A}-\langle\hat{A}\rangle_{\hat{\rho}} \hat{I}\right)^{2}\right\rangle_{\hat{\rho}}=\left\langle\hat{A}^{2}\right\rangle_{\hat{\rho}}-\left(\langle\hat{A}\rangle_{\hat{\rho}}\right)^{2} .
$$

Unless $\lambda \mu=1$, the operators (1.1) are not genuine EPR observables since they do not commute. In Refs. [8, 9], a 
possible experimental observation of the inequality

$$
\Delta Q(\lambda) \Delta P(\mu)<\frac{1}{2}
$$

is interpreted as a sufficient condition to detect an EPR paradox. It is interesting to remark that the EPR paradox 7] as shown in Eq. (1.4) and the concept of steering introduced by Schrödinger [10] as analyzed in Refs. 1113] were proven to be equivalent descriptions of nonlocality. Moreover, the EPR-steering turned out to be a different kind of non-locality stronger than quantum inseparability [11, 12].

An important piece of progress in studying separability starting with the uncertainty principle was made by Duan et al. [14]. They have introduced another family of EPR-like uncertainties in terms of the variances of the non-local one-parameter operators:

$\hat{Q}(\alpha):=\alpha \hat{q}_{1}-\frac{1}{\alpha} \hat{q}_{2}, \quad \hat{P}_{ \pm}(\alpha):=\alpha \hat{p}_{1} \pm \frac{1}{\alpha} \hat{p}_{2}$,

The commutation relations

$$
\left[\hat{Q}(\alpha), \hat{P}_{ \pm}(\alpha)\right]=i\left(\alpha^{2} \mp \frac{1}{\alpha^{2}}\right) \hat{I}
$$

lead to the product-form uncertainty relations for the EPR-like observables (1.5),

$$
\Delta Q(\alpha) \Delta P_{ \pm}(\alpha) \geqq \frac{1}{2}\left|\alpha^{2} \mp \frac{1}{\alpha^{2}}\right|,
$$

which imply the sum-form inequalities:

$$
[\Delta Q(\alpha)]^{2}+\left[\Delta P_{ \pm}(\alpha)\right]^{2} \geqq\left|\alpha^{2} \mp \frac{1}{\alpha^{2}}\right| .
$$

In Ref. [14], the inequalities (1.8) are strengthen for separable states: a necessary condition of separability, consisting of one-parameter family of inequalities, is thereby established for any two-mode state. Moreover, for the special class of TMGSs, the strongest of these inequalities is proven to be also a sufficient condition of separability. Some other necessary conditions of separability which employ pairs of more general non-local observables depending on one or more parameters have been pointed out [3, 15, 16]. They are expressed in terms of the product or the sum of variances of such EPR-like observables.

The aim of this paper is twofold. On the one hand, we tackle a characterization of the separability of TMGSs based on the analysis of EPR-like correlations. We write full criteria of separability for TMGSs in terms of both the product and the sum of variances of two EPR-like observables. Their formulation requires an explicit use of Simon's PPT criterion of separability. On the other hand, we offer a fresh look at the work of Duan et al. 14], which is based on an EPR-like uncertainty of sum form and is fully independent of the PPT approach. First, a suitable optimization of this EPR-like uncertainty sum leads eventually to an EPR-like indicator of separability for TMGSs, which is directly related to a specific classicality condition in the quantum optical sense. Second, we prove that the corresponding condition of separability is manifestly equivalent to that obtained by Simon in the framework of the PPT approach. This is an important aspect of the Gaussian separability problem which remained unclear for many years and was falsely doubted quite recently [17, 18].

The article is structured in the following way. In Sec. II, we recall the EPR-like necessary conditions of separability for two-mode states, as discussed by Giovannetti et al. in Ref. [16]. Section III is an overview of several useful properties of the undisplaced TMGSs. In Sec. IV, the product function occurring in Eq. (1.3) and one of the sum functions from Eq. (1.8) are normalized according to the formulae presented in Sec. II. Here we evaluate their minimal values over the whole range of the parameters defining the EPR-like observables, as well as under local unitary actions. We thus find the maximal EPR-like correlations over the family of equally entangled states. A two-parameter regularized sum function is examined along the same lines in Sec. V, where its minimum is found. On the one hand, the formulae established in Secs. IV and V allow us to derive the Peres-Simon PPT necessary condition of separability for a TMGS from each of the three EPR-like necessary conditions we have employed. On the other hand, they enable us to formulate full criteria of separability for TMGSs, owing to the PPT criterion of separability [3]. In Subsec. VI A, we describe the optimization of the regularized EPR-like uncertainty sum introduced by Duan et al. [14]. This leads to the socalled standard form II of the covariance matrix (CM), whose existence is proven in Subsec. VI B. Then, in Subsec. VI C, we build an EPR-like separability indicator in terms of the standard form II of the CM. Subsection VI D is devoted to some special cases when the EPR-like separability indicator can be determined explicitly. In Sec. VII, we establish directly the equivalence of the EPRlike and PPT conditions of separability. Section VIII summarizes our main results insisting on the connection between the EPR-like and PPT separability conditions for a TMGS. Appendix A presents some nontrivial identities involving the standard-form parameters of the CM of a TMGS. In Appendix B, we prove the positivity of the Hessian matrices of the three EPR-like correlation functions examined in Secs. IV and V, when evaluated at their stationary points.

\section{SEPARABLE TWO-MODE STATES}

Let us consider a pair of EPR-like observables which are linear combinations of the canonical quadrature operators of the two modes:

$$
\begin{aligned}
& \hat{Q}:=\alpha_{1} \hat{q}_{1}-\alpha_{2} \hat{q}_{2}, \quad \hat{P}:=\beta_{1} \hat{p}_{1}+\beta_{2} \hat{p}_{2}, \\
& \left(\alpha_{j}>0, \quad \beta_{j}>0: j=1,2\right) .
\end{aligned}
$$


The coordinates and momenta in Eq. (2.1) are defined in terms of the amplitude operators of the modes:

$$
\begin{aligned}
& \hat{q}_{j}:=\frac{1}{\sqrt{2}}\left(\hat{a}_{j}+\hat{a}_{j}^{\dagger}\right), \quad \hat{p}_{j}:=\frac{1}{\sqrt{2} i}\left(\hat{a}_{j}-\hat{a}_{j}^{\dagger}\right), \\
& (j=1,2) .
\end{aligned}
$$

Obviously, Reid's pair of one-parameter observables built with independent parameters (1.1), as well as the singleparameter one (1.5) are particular cases of EPR-like operators (2.1). The commutation relation

$$
[\hat{Q}, \hat{P}]=i\left(\alpha_{1} \beta_{1}-\alpha_{2} \beta_{2}\right) \hat{I}
$$

shows that the operators (2.1) are genuine EPR observables if and only if $\alpha_{1} \beta_{1}=\alpha_{2} \beta_{2}$. The corresponding Heisenberg uncertainty relation,

$$
\Delta Q \Delta P \geqq \frac{1}{2}\left|\alpha_{1} \beta_{1}-\alpha_{2} \beta_{2}\right|,
$$

entails the sum-type inequality

$$
(\Delta Q)^{2}+(\Delta P)^{2} \geqq\left|\alpha_{1} \beta_{1}-\alpha_{2} \beta_{2}\right| .
$$

If the two-mode state is separable, i. e., it is a convex combination of product states,

$$
\hat{\rho}_{s}:=\sum_{k} w_{k} \hat{\rho}_{1}^{(k)} \otimes \hat{\rho}_{2}^{(k)}, \quad\left(w_{k}>0, \quad \sum_{k} w_{k}=1\right),
$$

then the product $(\Delta Q)_{s}(\Delta P)_{s}$ has a stronger lower bound than in Eq. (2.4) [16]:

$$
(\Delta Q)_{s}(\Delta P)_{s} \geqq \frac{1}{2}\left(\alpha_{1} \beta_{1}+\alpha_{2} \beta_{2}\right) .
$$

Accordingly, for the sum of variances, an inequality stronger than Eq. (2.5) holds:

$$
\left[(\Delta Q)_{s}\right]^{2}+\left[(\Delta P)_{s}\right]^{2} \geqq \alpha_{1} \beta_{1}+\alpha_{2} \beta_{2} .
$$

It is useful to specialize the necessary conditions for the separability of a two-mode state, Eqs. (2.6) and (2.7), to the pairs of EPR-like observables (1.1) and (1.5). We get the following two sets of inequalities:

$$
\begin{array}{r}
{[\Delta Q(\lambda)]_{s}[\Delta P(\mu)]_{s} \geqq \frac{1}{2}(1+\lambda \mu),} \\
\left\{[\Delta Q(\lambda)]_{s}\right\}^{2}+\left\{[\Delta P(\mu)]_{s}\right\}^{2} \geqq 1+\lambda \mu ; \\
{[\Delta Q(\alpha)]_{s}\left[\Delta P_{ \pm}(\alpha)\right]_{s} \geqq \frac{1}{2}\left(\alpha^{2}+\frac{1}{\alpha^{2}}\right),} \\
\left\{[\Delta Q(\alpha)]_{s}\right\}^{2}+\left\{\left[\Delta P_{ \pm}(\alpha)\right]_{s}\right\}^{2} \geqq \alpha^{2}+\frac{1}{\alpha^{2}} .
\end{array}
$$

The necessary conditions of separability (2.11) have first been derived in Ref. [14].Following a similar pattern, the product-form conditions (2.8) and (2.10) have then been written in Refs. 15, 16]. Interestingly enough, it was shown in Ref. [16] that the product-form necessary conditions of separability are stronger than the corresponding sum-form ones. We also remark that some particular forms of the necessary conditions of separability (2.8) (2.11) have been used to detect entanglement in experiments [19 21].

\section{TWO-MODE GAUSSIAN STATES}

For a long time, the Gaussian states (GSs) of the quantum radiation field are known to be of central importance in various areas of quantum optics. In general, the GSs play an important role for those quantum systems involving a quadratic bosonic Hamiltonian that generates correlations between bosonic modes. They are achieved in condensed matter, as well as in atomic ensembles such as trapped ions or Bose-Einstein condensates. The GSs of light are also largely employed in quantum information processing with continuous variables; their usefulness has been reviewed in Refs. 22 24.

Especially accessible and insightful are the TMGSs. They have a privileged position among the bipartite quantum states of continuous-variable systems. Specifically, their properties are fully determined by the firstand second-order moments of the quadrature observables of the modes [25].

From the theoretical point of view, the TMGSs represent a perfect test bed for studying entanglement or other kinds of correlations between the modes [26]. Note that the entanglement of a TMGS depends only on its CM, so that it can be checked and quantified easier than for other two-party states. Here we recollect just the strictly necessary notions and results [25, 27, 28] that enable us to discuss separability issues. Since these are not affected by translations in the phase space, it is sufficient to deal with undisplaced (zero-mean) TMGSs.

Recall that the characteristic function $(\mathrm{CF})$ of an undisplaced TMGS $\hat{\rho}$ is a real exponential:

$$
\chi(u)=\exp \left[-\frac{1}{2}(J u)^{T} \mathcal{V}(J u)\right] .
$$

Its argument is a dimensionless vector $u \in \mathbb{R}^{4}$ whose components are eigenvalues of the canonical quadrature operators of the modes:

$$
u^{T}:=\left(q_{1}, p_{1}, q_{2}, p_{2}\right) .
$$

$J$ designates the standard matrix of the symplectic form on $\mathbb{R}^{4}$, which is block-diagonal and skew-symmetric:

$$
J:=J_{1} \oplus J_{2}: \quad J_{1}=J_{2}:=\left(\begin{array}{cc}
0 & 1 \\
-1 & 0
\end{array}\right) .
$$


The TMGS $\hat{\rho}$ is entirely specified, via the CF (3.1), by the real and symmetric $4 \times 4 \mathrm{CM}$, which is denoted $\mathcal{V}$. Its entries are expectation values of products of the deviations from the means of the quadratures (2.2). The order of the rows and columns is indicated by the current vector (3.2). The CM $\mathcal{V}$ fulfills the strong form (RobertsonSchrödinger) of the uncertainty relations for the canonical quadrature observables (2.2):

$$
\mathcal{V}+\frac{i}{2} J \geqslant 0
$$

The above requirement that the complex matrix $\mathcal{V}+\frac{i}{2} J$ has to be positive semidefinite is a necessary and sufficient condition for the existence of the GS $\hat{\rho}[3]$. It implies the inequality

$$
\mathcal{D}:=\operatorname{det}\left(\mathcal{V}+\frac{i}{2} J\right) \geqq 0,
$$

as well as the general property that the $\mathrm{CM} \mathcal{V}$ is positive definite: $\mathcal{V}>0$. By contrast, the saturation equality $\mathcal{D}=0$ is a quite special feature. However, it is shared by all the pure GSs and also by an interesting class of mixed ones. All these states are said to be at the physicality edge.

It is often convenient to partition the $\mathrm{CM} \mathcal{V}$ into $2 \times 2$ submatrices:

$$
\mathcal{V}=\left(\begin{array}{ll}
\mathcal{V}_{1} & \mathcal{C} \\
\mathcal{C}^{T} & \mathcal{V}_{2}
\end{array}\right)
$$

Here $\mathcal{V}_{j}, \quad(j=1,2)$, denote the $\mathrm{CMs}$ of the individual single-mode reduced GSs, while $\mathcal{C}$ displays the crosscorrelations between the modes.

Let us mention that a symplectic transformation $S$ of the canonical quadrature observables (2.2) in the Heisenberg picture induces a congruence transformation of any CM:

$$
\mathcal{V}^{\prime}=S \mathcal{V} S^{T}, \quad S \in \operatorname{Sp}(4, \mathbb{R})
$$

At the same time, a unitary operator $\hat{U}(S)$ acting on the two-mode Fock space $\mathcal{H}_{1} \otimes \mathcal{H}_{2}$ is associated to the symplectic matrix $S$. Remarkably, the corresponding transformation of the TMGS in the Schrödinger picture, $\hat{\rho}^{\prime}=\hat{U}(S) \hat{\rho} \hat{U}^{\dagger}(S)$, preserves the Gaussian nature of the state. Note that, if the symplectic transformation $S$ consists of two separate single-mode ones,

$$
S=S_{1} \oplus S_{2}: \quad S \in \operatorname{Sp}(2, \mathbb{R}) \times \operatorname{Sp}(2, \mathbb{R})
$$

then the above transformation of the TMGS $\hat{\rho}$ does not affect its amount of entanglement. It has been proven in Ref. [14] and just exploited in Ref. [3] the existence of a local symplectic transformation (3.8) that leads to a standard form of the CM:

$$
\mathcal{V}(1,1):=\left(\begin{array}{cccc}
b_{1} & 0 & c & 0 \\
0 & b_{1} & 0 & d \\
c & 0 & b_{2} & 0 \\
0 & d & 0 & b_{2}
\end{array}\right),
$$

depending on four parameters $b_{1}, b_{2}, c, d$. With no loss of generality, one can choose $b_{1} \geqq b_{2}>0$ and $c \geqq|d|$. Following Refs. [3, 14], we apply two independent onemode squeeze transformations to the standard-form CM (3.9). Let us denote the corresponding scaling factors by

$$
u_{1}:=e^{2 r_{1}} \geqq 1, \quad u_{2}:=e^{2 r_{2}} \geqq 1,
$$

where $r_{1}$ and $r_{2}$ are the squeeze parameters in the two modes. The transformed state has a scaled standard-form CM with the block structure (3.6):

$$
\mathcal{V}\left(u_{1}, u_{2}\right)=\left(\begin{array}{cc}
\mathcal{V}_{1}\left(u_{1}\right) & \mathcal{C}\left(\sqrt{u_{1} u_{2}}\right) \\
\mathcal{C}\left(\sqrt{u_{1} u_{2}}\right) & \mathcal{V}_{2}\left(u_{2}\right)
\end{array}\right)
$$

All its $2 \times 2$ submatrices are diagonal. The CMs of the single-mode reduced GSs read

$$
\mathcal{V}_{j}\left(u_{j}\right)=\left(\begin{array}{cc}
b_{j} u_{j} & 0 \\
0 & \frac{b_{j}}{u_{j}}
\end{array}\right), \quad(j=1,2),
$$

while the cross-correlation matrix is

$$
\mathcal{C}\left(\sqrt{u_{1} u_{2}}\right)=\left(\begin{array}{cc}
c \sqrt{u_{1} u_{2}} & 0 \\
0 & \frac{d}{\sqrt{u_{1} u_{2}}}
\end{array}\right) .
$$

The TMGSs whose CMs have a scaled standard form (3.11) - (3.13) constitute a class characterized by the set of four standard-form parameters $\left\{b_{1}, b_{2}, c, d\right\}$ of the given TMGS $\hat{\rho}$, which are $\operatorname{Sp}(2, \mathbb{R}) \times \operatorname{Sp}(2, \mathbb{R})$-invariant. The states of this class are labeled by the pair of local squeeze factors $\left\{u_{1}, u_{2}\right\}$ [14]. They are locally unitary similar to the given TMGS $\hat{\rho}$ and thereby possess precisely its amount of entanglement.

The Robertson-Schrödinger uncertainty relation 3.4 reduces to the following restrictions for the standard-form parameters:

$$
b_{1} \geqq \frac{1}{2}, \quad b_{2} \geqq \frac{1}{2} ;
$$

$$
\begin{aligned}
& b_{1} b_{2}-c^{2} \geqq \frac{1}{4} \max \left\{\frac{b_{1}}{b_{2}}, \frac{b_{2}}{b_{1}}\right\} \geqq \frac{1}{4}, \\
& b_{1} b_{2}-d^{2} \geqq \frac{1}{4} \max \left\{\frac{b_{1}}{b_{2}}, \frac{b_{2}}{b_{1}}\right\} \geqq \frac{1}{4}
\end{aligned}
$$




$$
\begin{aligned}
& \mathcal{D}=\left(b_{1} b_{2}-c^{2}\right)\left(b_{1} b_{2}-d^{2}\right) \\
& -\frac{1}{4}\left(b_{1}^{2}+b_{2}^{2}+2 c d\right)+\frac{1}{16} \geqq 0 .
\end{aligned}
$$

The inequalities (3.15) give rise to another one involving the symplectic invariant $\operatorname{det}(\mathcal{V})=\left(b_{1} b_{2}-c^{2}\right)\left(b_{1} b_{2}-d^{2}\right)$ and thus concerning the purity of the state:

$$
\operatorname{det}(\mathcal{V}) \geqq \frac{1}{16} \Longleftrightarrow \operatorname{Tr}\left(\hat{\rho}^{2}\right) \leqq 1
$$

According to Williamson's theorem [29], the CM $\mathcal{V}$ of any TMGS $\hat{\rho}$ is congruent to a diagonal CM via a symplectic matrix (3.7), which is unique up to the sign. The corresponding diagonal entries, denoted $\kappa_{ \pm}$, are positive, each one occurring twice. They are called the symplectic eigenvalues of the $\mathrm{CM} \mathcal{V}[30]$. By virtue of Eq. (3.7), the symplectic invariants $\operatorname{det}(\mathcal{V})$ and $\mathcal{D}$ factor as follows:

$$
\begin{gathered}
\operatorname{det}(\mathcal{V})=\left(\kappa_{+}\right)^{2}\left(\kappa_{-}\right)^{2}, \\
\mathcal{D}=\left[\left(\kappa_{+}\right)^{2}-\frac{1}{4}\right]\left[\left(\kappa_{-}\right)^{2}-\frac{1}{4}\right] \geqq 0,
\end{gathered}
$$

with

$$
\kappa_{+} \geqq \kappa_{-} \geqq \frac{1}{2}
$$

We employ Eqs. (3.18) and (3.19) to get the symplectic eigenvalues in terms of the standard-form parameters [30]:

$$
\begin{aligned}
& \left(\kappa_{ \pm}\right)^{2}=\frac{1}{2}\left[\left(b_{1}^{2}+b_{2}^{2}+2 c d\right) \pm \sqrt{\Delta}\right], \\
& \Delta:=\left(b_{1}^{2}+b_{2}^{2}+2 c d\right)^{2}-4 \operatorname{det}(\mathcal{V}) \\
& =\left(b_{1}^{2}-b_{2}^{2}\right)^{2}+4\left(b_{1} c+b_{2} d\right)\left(b_{2} c+b_{1} d\right) \geqq 0 .
\end{aligned}
$$

In order to formulate Simon's separability criterion for TMGSs, we have to consider the partial transpose $\hat{\rho}^{\mathrm{PT}}$ of the TMGS $\hat{\rho}$. Basically, partial transposition preserves the Gaussian character of the operator, does not modify the standard-form parameters $b_{1}, b_{2}, c$, and changes the sign of $d$. Therefore, $\hat{\rho}^{\mathrm{PT}}$ is an undisplaced two-mode Gaussian operator whose $\mathrm{CM} \mathcal{V}^{\mathrm{PT}}$ has the standard-form parameters $\left\{b_{1}, b_{2}, c,-d\right\}[3]$. First, Simon has proven a lemma asserting that any TMGS with $d \geqq 0$ is separable. Then, he has shown that Peres' necessary condition of separability, which claims that $\hat{\rho}^{\mathrm{PT}}$ should be a quantum state, i. e.,

$$
\mathcal{V}^{\mathrm{PT}}+\frac{i}{2} J \geqslant 0
$$

is also a sufficient one [3]. The condition (3.22) for the existence of a GS $\hat{\rho}^{\mathrm{PT}}$ reduces to the inequality

$$
\mathcal{D}^{\mathrm{PT}}:=\operatorname{det}\left(\mathcal{V}^{\mathrm{PT}}+\frac{i}{2} J\right) \geqq 0,
$$

which is Simon's criterion of separability. It is usually written in the form (3.16) with $d \rightarrow-d$. Therefore, a TMGS is separable if and only if the following inequality is fulfilled:

$$
\mathcal{D}^{\mathrm{PT}}=\operatorname{det}(\mathcal{V})-\frac{1}{4}\left(b_{1}^{2}+b_{2}^{2}-2 c d\right)+\frac{1}{16} \geqq 0 .
$$

Accordingly, the identity

$$
\mathcal{D}^{\mathrm{PT}}=\mathcal{D}+c d
$$

displays the property that all the TMGSs with $d \geqq 0$ are separable, in agreement with Simon's lemma [3].

\section{NORMALIZED SEPARABILITY INDICATORS}

We write the variances of the EPR-like observables (2.1) for a TMGS whose CM has a scaled standard form (3.11) - 3.13):

$$
(\Delta Q)^{2}=\alpha_{1}^{2} b_{1} u_{1}+\alpha_{2}^{2} b_{2} u_{2}-2 \alpha_{1} \alpha_{2} c \sqrt{u_{1} u_{2}},
$$

$$
(\Delta P)^{2}=\beta_{1}^{2} \frac{b_{1}}{u_{1}}+\beta_{2}^{2} \frac{b_{2}}{u_{2}}+2 \beta_{1} \beta_{2} \frac{d}{\sqrt{u_{1} u_{2}}} .
$$

Remark that, while all the TMGSs (3.11)- 3.13) belonging to a class with fixed standard-form parameters possess the same amount of entanglement, their EPRlike variances (4.1) and (4.2) depend, in addition, on the local squeezings $u_{1}, u_{2}$. In what follows, we consider two functions which are normalized as suggested by the separability lower bounds (2.6) and (2.7):

$$
\begin{gathered}
E\left(\alpha_{1} \beta_{1}, \alpha_{2}, \beta_{2}, u_{1}, u_{2}\right):=\frac{(\Delta Q)^{2}(\Delta P)^{2}}{\left(\alpha_{1} \beta_{1}+\alpha_{2} \beta_{2}\right)^{2}}, \\
F\left(\alpha_{1}, \beta_{1}, \alpha_{2}, \beta_{2}, u_{1}, u_{2}\right):=\frac{(\Delta Q)^{2}+(\Delta P)^{2}}{\alpha_{1} \beta_{1}+\alpha_{2} \beta_{2}} .
\end{gathered}
$$

According to Eqs. (2.6) and (2.7), for any separable twomode state (Gaussian or non-Gaussian), the following inequalities are satisfied:

$$
E \geqq \frac{1}{4}, \quad F \geqq 1 .
$$

In the sequel, we develop a two-step program. First, in order to handle simpler functions, we diminish the number of their independent variables as much as possible by making suitable substitutions or choices. Second, we find the absolute minima of the resulting simpler functions, which prove to be manifest separability indicators. It is sufficient to restrict our search in this step to values $d<0$ in the variance (4.2). 


\section{A. Uncertainty product}

We start by absorbing the scaling factors in Eqs. 4.1 and (4.2) into four new positive parameters,

$$
\alpha_{j}^{\prime}=\alpha_{j} \sqrt{u_{j}}, \quad \beta_{j}^{\prime}=\beta_{j} \frac{1}{\sqrt{u_{j}}}, \quad(j=1,2),
$$

so that the variances become:

$$
\begin{aligned}
& (\Delta Q)^{2}=b_{1}\left(\alpha_{1}^{\prime}\right)^{2}+b_{2}\left(\alpha_{2}^{\prime}\right)^{2}-2 c \alpha_{1}^{\prime} \alpha_{2}^{\prime}, \\
& (\Delta P)^{2}=b_{1}\left(\beta_{1}^{\prime}\right)^{2}+b_{2}\left(\beta_{2}^{\prime}\right)^{2}+2 d \beta_{1}^{\prime} \beta_{2}^{\prime} .
\end{aligned}
$$

Note the identity

$$
\alpha_{1} \beta_{1}+\alpha_{2} \beta_{2}=\alpha_{1}^{\prime} \beta_{1}^{\prime}+\alpha_{2}^{\prime} \beta_{2}^{\prime} .
$$

After replacing Eqs. (4.7)- (4.9) into Eq. (4.3), the function $E\left(\alpha_{1}^{\prime} \beta_{1}^{\prime}, \alpha_{2}^{\prime}, \beta_{2}^{\prime}\right)$ can be further simplified. As a matter of fact, it is a function of two positive variables,

$$
\lambda:=\frac{\alpha_{2}^{\prime}}{\alpha_{1}^{\prime}}, \quad \mu:=\frac{\beta_{2}^{\prime}}{\beta_{1}^{\prime}},
$$

which reads:

$$
E(\lambda, \mu):=\frac{[\Delta Q(\lambda)]^{2}[\Delta P(\mu)]^{2}}{(1+\lambda \mu)^{2}} .
$$

The numerator of the fraction in the r. h. s. of Eq. (4.11) is equal to the product of the variances of Reid's EPR-like observables (1.1) in a standard-form TMGS (3.9):

$$
\begin{aligned}
& {[\Delta Q(\lambda)]^{2}=b_{1}+b_{2} \lambda^{2}-2 c \lambda,} \\
& {[\Delta P(\mu)]^{2}=b_{1}+b_{2} \mu^{2}+2 d \mu .}
\end{aligned}
$$

We prove the following statement.

Theorem 1. For any TMGS with $d<0$ the positive function $E(\lambda, \mu)$ has an absolute minimum equal to the square of the smallest symplectic eigenvalue $\kappa_{-}^{\mathrm{PT}}$ :

$$
E_{m}:=\min _{\{\lambda, \mu\}} E(\lambda, \mu)=\left(\kappa_{-}^{\mathrm{PT}}\right)^{2} .
$$

Proof. The first-order derivatives

$$
\begin{aligned}
& \frac{\partial \ln (E)}{\partial \lambda}=\frac{2\left(b_{2} \lambda-c\right)}{b_{1}+b_{2} \lambda^{2}-2 c \lambda}-\frac{2 \mu}{1+\lambda \mu}, \\
& \frac{\partial \ln (E)}{\partial \mu}=\frac{2\left(b_{2} \mu+d\right)}{b_{1}+b_{2} \mu^{2}+2 d \mu}-\frac{2 \lambda}{1+\lambda \mu}
\end{aligned}
$$

vanish at a stationary point of the function (4.11). The resulting equations,

$$
\begin{aligned}
& {[\Delta Q(\lambda)]^{2}=\frac{1}{\mu}\left(b_{2} \lambda-c\right)(1+\lambda \mu),} \\
& {[\Delta P(\mu)]^{2}=\frac{1}{\lambda}\left(b_{2} \mu+d\right)(1+\lambda \mu),}
\end{aligned}
$$

have a unique solution:

$$
\lambda_{m}=\frac{\left(b_{1}^{2}-b_{2}^{2}\right)+\sqrt{\Delta^{\mathrm{PT}}}}{2\left(b_{1} c-b_{2} d\right)}, \quad \mu_{m}=\frac{\left(b_{1}^{2}-b_{2}^{2}\right)+\sqrt{\Delta^{\mathrm{PT}}}}{2\left(b_{2} c-b_{1} d\right)},
$$

where $\Delta^{\mathrm{PT}}$ is the discriminant in Eq. A5 . In Appendix $\mathrm{B}$ we have proven that the value (B4),

$$
E_{m}=\frac{\left(b_{2} \lambda_{m}-c\right)\left(b_{2} \mu_{m}+d\right)}{\lambda_{m} \mu_{m}},
$$

is the absolute minimum of the function $E(\lambda, \mu)$. By use of Eqs. 4.17) and (A5), we find the formula

$$
E_{m}=\left(\kappa_{-}^{\mathrm{PT}}\right)^{2} \text {. }
$$

Therefore, the minimum of the normalized product (4.11) of two EPR-like uncertainties is equal to the square of the smallest symplectic eigenvalue $\kappa_{-}^{\mathrm{PT}}$. This concludes the proof. Note that although stated for $d<0$, Eq. (4.19) still holds for $0 \leqq d<\frac{b_{2}}{b_{1}} c$.

A previous relationship between $\kappa_{-}^{\mathrm{PT}}$ and a product of EPR-like uncertainties was found by a different treatment and from another perspective in Ref. 31. The normalized product of the EPR-like uncertainties (4.12) and (4.13) was recently examined as a signature of entanglement for special families of TMGSs 32 34 . The present optimization approach was successfully applied in Ref. 34] to the class of the squeezed thermal states (STSs): $c=-d>0$ [27].

An interesting related problem for TMGSs regarding steerability criteria was recently put forward by Kogias and Adesso [35]. These authors have considered the product of EPR-like uncertainties occurring in the Reid condition (1.4) and have minimized it with respect to the pair of parameters $\{\lambda, \mu\}$ and the local variables of a TMGS. They have thus recovered the explicit symplectically invariant formula for the condition of steerability of GSs by Gaussian measurements which has first been written in Refs. [11, 12].

\section{B. Uncertainty sum}

We find it convenient to simplify the function (4.4) by setting $\alpha_{1}=\beta_{1}=: \alpha, \alpha_{2}=\beta_{2}=: \frac{1}{\alpha}$ :

$$
F\left(\alpha^{2}, u_{1}, u_{2}\right):=\frac{[\Delta Q(\alpha)]^{2}+\left[\Delta P_{+}(\alpha)\right]^{2}}{\alpha^{2}+\frac{1}{\alpha^{2}}} .
$$

The correlation function (4.20) is built with the variances of a pair of non-local one-parameter observables (1.5) introduced in Ref. [14]:

$$
[\Delta Q(\alpha)]^{2}=b_{1} u_{1} \alpha^{2}+b_{2} u_{2} \frac{1}{\alpha^{2}}-2 c \sqrt{u_{1} u_{2}},
$$




$$
\left[\Delta P_{ \pm}(\alpha)\right]^{2}=\frac{b_{1}}{u_{1}} \alpha^{2}+\frac{b_{2}}{u_{2}} \frac{1}{\alpha^{2}} \pm \frac{2 d}{\sqrt{u_{1} u_{2}}} .
$$

Therefore, it reads:

$$
\begin{aligned}
& F\left(\alpha^{2}, u_{1}, u_{2}\right)=\frac{1}{\alpha^{4}+1}\left[b_{1}\left(u_{1}+\frac{1}{u_{1}}\right) \alpha^{4}-2\left(c \sqrt{u_{1} u_{2}}\right.\right. \\
& \left.\left.-d \frac{1}{\sqrt{u_{1} u_{2}}}\right) \alpha^{2}+b_{2}\left(u_{2}+\frac{1}{u_{2}}\right)\right] .
\end{aligned}
$$

\section{We state}

Theorem 2. For any TMGS with $d<0$ the positive function $F\left(\alpha^{2}, u_{1}, u_{2}\right)$ has an absolute minimum which is twice the smallest symplectic eigenvalue $\kappa_{-}^{\mathrm{PT}}$ :

$$
F_{m}:=\min _{\left\{\alpha^{2}, u_{1}, u_{2}\right\}} F\left(\alpha^{2}, u_{1}, u_{2}\right)=2 \kappa_{-}^{\mathrm{PT}} .
$$

Proof. The first-order derivatives

$$
\begin{gathered}
\frac{\partial F}{\partial\left(\alpha^{2}\right)}=\frac{2}{\left(\alpha^{4}+1\right)^{2}}\left\{\left(c \sqrt{u_{1} u_{2}}-\frac{d}{\sqrt{u_{1} u_{2}}}\right)\left(\alpha^{4}-1\right)\right. \\
\left.+\left[b_{1}\left(u_{1}+\frac{1}{u_{1}}\right)-b_{2}\left(u_{2}+\frac{1}{u_{2}}\right)\right] \alpha^{2}\right\} \\
\frac{\partial F}{\partial u_{1}}=\frac{\alpha^{2}}{\left(\alpha^{4}+1\right) u_{1}}\left[b_{1}\left(u_{1}-\frac{1}{u_{1}}\right) \alpha^{2}\right. \\
\left.-\left(c \sqrt{u_{1} u_{2}}+\frac{d}{\sqrt{u_{1} u_{2}}}\right)\right], \\
\frac{\partial F}{\partial u_{2}}=\frac{\alpha^{2}}{\left(\alpha^{4}+1\right) u_{2}}\left[b_{2}\left(u_{2}-\frac{1}{u_{2}}\right) \frac{1}{\alpha^{2}}\right. \\
\left.-\left(c \sqrt{u_{1} u_{2}}+\frac{d}{\sqrt{u_{1} u_{2}}}\right)\right]
\end{gathered}
$$

vanish at a stationary point of the function (4.23). We try to solve the resulting system of stationarity equations:

$$
\begin{aligned}
& \left(c \sqrt{u_{1} u_{2}}-\frac{d}{\sqrt{u_{1} u_{2}}}\right)\left(1-\alpha^{4}\right) \\
& =\left[b_{1}\left(u_{1}+\frac{1}{u_{1}}\right)-b_{2}\left(u_{2}+\frac{1}{u_{2}}\right)\right] \alpha^{2}, \\
& b_{1}\left(u_{1}-\frac{1}{u_{1}}\right) \alpha^{2}=c \sqrt{u_{1} u_{2}}+\frac{d}{\sqrt{u_{1} u_{2}}}, \\
& b_{2}\left(u_{2}-\frac{1}{u_{2}}\right) \frac{1}{\alpha^{2}}=c \sqrt{u_{1} u_{2}}+\frac{d}{\sqrt{u_{1} u_{2}}} .
\end{aligned}
$$

From Eqs. (4.29) and (4.30) it follows:

$$
\alpha^{4}=\frac{b_{2}\left(u_{2}-\frac{1}{u_{2}}\right)}{b_{1}\left(u_{1}-\frac{1}{u_{1}}\right)}
$$

$$
\begin{gathered}
b_{1} b_{2}\left(u_{1}-\frac{1}{u_{1}}\right)\left(u_{2}-\frac{1}{u_{2}}\right) \\
=\left(c \sqrt{u_{1} u_{2}}+\frac{d}{\sqrt{u_{1} u_{2}}}\right)^{2} ; \\
{\left[\Delta P_{+}(\alpha)\right]^{2}=[\Delta Q(\alpha)]^{2} .}
\end{gathered}
$$

Insertion of Eqs. (4.29) and (4.31) into Eq. (4.28) gives the proportionality relation

$$
u_{2}=\gamma u_{1}, \quad \gamma:=\frac{b_{2} c-b_{1} d}{b_{1} c-b_{2} d} \leqq 1 .
$$

By substituting it into Eq. 4.32, we get a quadratic equation in the product $p:=u_{1} u_{2} \geqq 1$ :

$$
\begin{aligned}
& \left(b_{1} b_{2}-c^{2}\right) p^{2}-\left[b_{1} b_{2}\left(\gamma+\frac{1}{\gamma}\right)+2 c d\right] p \\
& +\left(b_{1} b_{2}-d^{2}\right)=0
\end{aligned}
$$

with

$$
\gamma+\frac{1}{\gamma}=2+\frac{\left[\left(b_{1}-b_{2}\right)(c+d)\right]^{2}}{\left(b_{1} c-b_{2} d\right)\left(b_{2} c-b_{1} d\right)} .
$$

Let us denote by $\Delta_{p}$ the discriminant of the quadratic trinomial in Eq. 4.35 and let $p_{ \pm}$be its roots. Making use of Eq. (A5), we find the relation

$$
\Delta_{p}=\left[\frac{b_{1} b_{2}\left(c^{2}-d^{2}\right)}{\left(b_{1} c-b_{2} d\right)\left(b_{2} c-b_{1} d\right)}\right]^{2} \Delta^{\mathrm{PT}} \geqq 0 .
$$

Since $p_{-}<1$ for $c+d>0$, the only acceptable solution of the quadratic equation 4.35) is

$$
p_{+}=\frac{c\left(b_{1} b_{2}-d^{2}\right)-d\left(\kappa_{-}^{\mathrm{PT}}\right)^{2}}{-d\left(b_{1} b_{2}-c^{2}\right)+c\left(\kappa_{-}^{\mathrm{PT}}\right)^{2}} \geqq 1 .
$$

With Eqs. (4.34) and (4.38) we get the scaling factors:

$$
\begin{aligned}
& u_{1 m}=\left[\frac{b_{2}\left(b_{1} b_{2}-d^{2}\right)-b_{1}\left(\kappa_{-}^{\mathrm{PT}}\right)^{2}}{b_{2}\left(b_{1} b_{2}-c^{2}\right)-b_{1}\left(\kappa_{-}^{\mathrm{PT}}\right)^{2}}\right]^{\frac{1}{2}}, \\
& u_{2 m}=\left[\frac{b_{1}\left(b_{1} b_{2}-d^{2}\right)-b_{2}\left(\kappa_{-}^{\mathrm{PT}}\right)^{2}}{b_{1}\left(b_{1} b_{2}-c^{2}\right)-b_{2}\left(\kappa_{-}^{\mathrm{PT}}\right)^{2}}\right]^{\frac{1}{2}} .
\end{aligned}
$$

In view of Eq. (A5), they have the alternative expressions:

$$
\begin{aligned}
& u_{1 m}=\left\{\frac{b_{1}\left[\sqrt{\Delta^{\mathrm{PT}}}-\left(b_{1}^{2}-b_{2}^{2}\right)\right]+2 d\left(b_{1} c-b_{2} d\right)}{b_{1}\left[\sqrt{\Delta^{\mathrm{PT}}}-\left(b_{1}^{2}-b_{2}^{2}\right)\right]-2 c\left(b_{2} c-b_{1} d\right)}\right\}^{\frac{1}{2}}, \\
& u_{2 m}=\left\{\frac{b_{2}\left[\sqrt{\Delta^{\mathrm{PT}}}+\left(b_{1}^{2}-b_{2}^{2}\right)\right]+2 d\left(b_{2} c-b_{1} d\right)}{b_{2}\left[\sqrt{\Delta^{\mathrm{PT}}}+\left(b_{1}^{2}-b_{2}^{2}\right)\right]-2 c\left(b_{1} c-b_{2} d\right)}\right\}^{\frac{1}{2}} .
\end{aligned}
$$


Insertion of the scaling factors (4.40) into Eq. (4.31) leads to the following value of the EPR-like parameter $\alpha$ :

$$
\alpha_{m}=\left[\frac{\sqrt{\Delta^{\mathrm{PT}}}-\left(b_{1}^{2}-b_{2}^{2}\right)}{\sqrt{\Delta^{\mathrm{PT}}}+\left(b_{1}^{2}-b_{2}^{2}\right)}\right]^{\frac{1}{4}} \leqq 1 .
$$

There is therefore a single stationary value of the function $F\left(\alpha^{2}, u_{1}, u_{2}\right)$, Eq. (4.23):

$$
F_{m}=F\left(\alpha_{m}^{2}, u_{1 m}, u_{2 m}\right) .
$$

In fact, this is its absolute minimum, as proven in Appendix B. With the solution (4.39) and (4.41), we take advantage of Eqs. (4.33), (A6), and (A8) to find the minimum value

$$
F_{m}=2 \kappa_{-}^{\mathrm{PT}} .
$$

This concludes the proof. Moreover, although stated for $d<0$, Eq. (4.43) is also true for $0 \leqq d<\frac{b_{2}}{b_{1}} c$.

It is worth mentioning that Eqs. (4.40) and (4.41), which specify the coordinates of the minimum point, become much simpler for two classes of TMGSs, namely, the STSs and the symmetric states. We find it convenient to write down here the corresponding formulae.

1. Two-mode STSs:

$$
\begin{aligned}
& c=-d>0 \Longleftrightarrow u_{1 m}=u_{2 m}=1: \quad \gamma=1 ; \\
& \sqrt{\Delta^{\mathrm{PT}}}=\left(b_{1}+b_{2}\right) \sqrt{\delta}, \quad \delta:=\left(b_{1}-b_{2}\right)^{2}+4 c^{2}>0: \\
& \left(\alpha_{m}\right)^{2}=\frac{1}{2 c}\left[\sqrt{\delta}-\left(b_{1}-b_{2}\right)\right] .
\end{aligned}
$$

\section{Symmetric TMGSs:}

$$
\begin{aligned}
& b_{1}=b_{2}=: b \Longrightarrow u_{1 m}=u_{2 m}=\sqrt{\frac{b+d}{b-c}}: \quad \gamma=1 ; \\
& \sqrt{\Delta^{\mathrm{PT}}}=2 b(c-d)>0, \quad \alpha_{m}=1 .
\end{aligned}
$$

It is interesting to notice that in Ref. 28] the minimal EPR-like uncertainty in sum form was explicitly evaluated for symmetric TMGSs. The authors have interpreted therein the smallest symplectic eigenvalue of the partially transposed state as a quantifier of the greatest amount of EPR-like correlations which can be created in a symmetric TMGS by means of local operations. Our present findings in the general case, Eq. (4.19) for the product form of the normalized EPR-like uncertainties, and Eq. (4.43) for the sum-form ones, appear to be consistent with this interpretation. Therefore, the local squeezing factors (4.40) determine the state having the maximal normalized EPR-like correlations among the whole set of equally entangled TMGSs.

\section{Separability}

Let us write the necessary conditions of separability (4.5) for a TMGS by using the functions (4.11) and
(4.20):

$$
E(\lambda, \mu) \geqq \frac{1}{4}, \quad F\left(\alpha^{2}, u_{1}, u_{2}\right) \geqq 1 .
$$

When $d<0$, these inequalities hold for any values of the above functions, including their minima (4.19) and (4.43), respectively:

$$
E_{m}=\left(\kappa_{-}^{\mathrm{PT}}\right)^{2} \geqq \frac{1}{4}, \quad F_{m}=2 \kappa_{-}^{\mathrm{PT}} \geqq 1 .
$$

In the opposite case, $d \geqq 0$, the identity (3.25) entails two equivalent inequalities:

$$
\kappa_{-}^{\mathrm{PT}} \geqq \frac{1}{2} \Longleftrightarrow \mathcal{D}^{\mathrm{PT}} \geqq 0 .
$$

Therefore, each EPR-like necessary condition of separability (4.46) for a TMGS implies the Peres-Simon inequality (3.24). We have reached this conclusion making no use of the PPT theory.

On the other hand, Simon's necessary condition of separability (3.24) is also a sufficient one for any TMGS [3]. When $d \geqq 0$, the inequalities (4.48) hold, so that the TMGS is separable. The only debatable case is therefore $d<0$. We apply the PPT criterium of separability (4.48) in conjunction with Eqs. (4.19) and (4.43) to get two EPR-like conditions:

$$
E_{m} \geqq \frac{1}{4}, \quad F_{m} \geqq 1 \Longleftrightarrow \hat{\rho} \text { separable, } \quad(d<0) .
$$

Equation (4.49) can be written alternatively:

$$
E_{m}<\frac{1}{4}, \quad F_{m}<1 \Longleftrightarrow \hat{\rho} \text { entangled, } \quad(d<0) .
$$

Accordingly, both minima $E_{m}$ and $F_{m}$ are themselves separability indicators. This means that each of the identities (4.46) is true if and only if the TMGS is separable.

\section{SEPARABILITY INDICATOR IN REGULARIZED SUM FORM}

\section{A. EPR-like correlation function}

Let us start from the Reid's pair of EPR-like observables (1.1). One gets their variances by setting $\alpha_{1}=1$, $\alpha_{2}=: \lambda, \beta_{1}=1, \beta_{2}=: \mu$ in Eqs. (4.1) and (4.2):

$$
\begin{gathered}
(\Delta Q)^{2}=b_{1} u_{1}+b_{2} u_{2} \lambda^{2}-2 c \sqrt{u_{1} u_{2}} \lambda, \\
(\Delta P)^{2}=\frac{b_{1}}{u_{1}}+\frac{b_{2}}{u_{2}} \mu^{2}+2 \frac{d}{\sqrt{u_{1} u_{2}}} \mu .
\end{gathered}
$$

It is convenient to absorb the scaling factor $u_{2}$ into a pair of new EPR-like parameters:

$$
\xi:=\sqrt{u_{2}} \lambda>0, \quad \eta:=\frac{1}{\sqrt{u_{2}}} \mu>0 .
$$


This simplifies the variances (5.1) and (5.2) as follows:

$$
\begin{gathered}
{[\Delta Q(\xi)]^{2}=b_{1} u_{1}+b_{2} \xi^{2}-2 c \sqrt{u_{1}} \xi} \\
{[\Delta P(\eta)]^{2}=\frac{b_{1}}{u_{1}}+b_{2} \eta^{2}+2 \frac{d}{\sqrt{u_{1}}} \eta .}
\end{gathered}
$$

We take inspiration from the separability condition (2.7) to build an EPR-like correlation function of the remaining independent variables $\xi, \eta, u_{1}$ :

$$
G\left(\xi, \eta, u_{1}\right):=[\Delta Q(\xi)]^{2}+[\Delta P(\eta)]^{2}-(1+\xi \eta) .
$$

The function (5.6), which is non-negative for any separable TMGS, has the explicit form:

$$
\begin{aligned}
& G\left(\xi, \eta, u_{1}\right)=\left(b_{1} u_{1}+b_{2} \xi^{2}-2 c \sqrt{u_{1}} \xi\right) \\
& +\left(\frac{b_{1}}{u_{1}}+b_{2} \eta^{2}+2 \frac{d}{\sqrt{u_{1}}} \eta\right)-(1+\xi \eta) .
\end{aligned}
$$

The following statement is true.

Theorem 3. For any TMGS with $d<0$ the function $G\left(\xi, \eta, u_{1}\right)$ has an absolute minimum,

$$
G_{m}:=\min _{\left\{\xi, \eta, u_{1}\right\}} G\left(\xi, \eta, u_{1}\right),
$$

which is the product of the determinant $\mathcal{D}^{\mathrm{PT}}$ and a positive factor.

Proof. Let us assume that $d<0$ in Eq. (5.7) and in the subsequent ones. We write the stationarity conditions:

$$
\begin{array}{ll}
\frac{\partial G}{\partial \xi}=0: & 2 b_{2} \xi-\eta=2 c \sqrt{u_{1}}, \\
\frac{\partial G}{\partial \eta}=0: & -\xi+2 b_{2} \eta=-2 \frac{d}{\sqrt{u_{1}}} \\
\frac{\partial G}{\partial u_{1}}=0: & c u_{1} \xi+d \eta=\frac{b_{1}}{\sqrt{u_{1}}}\left(u_{1}^{2}-1\right) .
\end{array}
$$

The system (5.9) is linear in the variables (5.3). By solving the first two equations with respect to $\xi$ and $\eta$ and then replacing the result into the third one, we find its unique solution:

$$
\begin{aligned}
& \xi_{m}=\frac{1}{b_{2}^{2}-\frac{1}{4}}\left(b_{2} c \sqrt{u_{1 m}}-\frac{1}{2} \frac{d}{\sqrt{u_{1 m}}}\right), \\
& \eta_{m}=\frac{1}{b_{2}^{2}-\frac{1}{4}}\left(\frac{1}{2} c \sqrt{u_{1 m}}-b_{2} \frac{d}{\sqrt{u_{1 m}}}\right), \\
& u_{1 m}=\left[\frac{b_{2}\left(b_{1} b_{2}-d^{2}\right)-\frac{1}{4} b_{1}}{b_{2}\left(b_{1} b_{2}-c^{2}\right)-\frac{1}{4} b_{1}}\right]^{\frac{1}{2}} .
\end{aligned}
$$

In Appendix B we have proven that the only stationary value of the function (5.7),

$$
G_{m}=G\left(\xi_{m}, \eta_{m}, u_{1 m}\right)
$$

is its absolute minimum. Insertion of the coordinates (5.10) into Eq. (5.7) and a subsequent straightforward calculation yields the formula

$$
\begin{aligned}
& G_{m}=\frac{1}{b_{2}^{2}-\frac{1}{4}}\left(2 \left\{\left[b_{2}\left(b_{1} b_{2}-c^{2}\right)-\frac{1}{4} b_{1}\right]\right.\right. \\
& \left.\left.\times\left[b_{2}\left(b_{1} b_{2}-d^{2}\right)-\frac{1}{4} b_{1}\right]\right\}^{\frac{1}{2}}-\left(b_{2}^{2}-\frac{1}{4}-c d\right)\right) .
\end{aligned}
$$

Making use of the identity (A10), the minimum (5.12) can be cast into the form:

$$
\begin{aligned}
& G_{m}=4 \mathcal{D}^{\mathrm{PT}}\left(2 \left\{\left[b_{2}\left(b_{1} b_{2}-c^{2}\right)-\frac{1}{4} b_{1}\right]\right.\right. \\
& \left.\left.\times\left[b_{2}\left(b_{1} b_{2}-d^{2}\right)-\frac{1}{4} b_{1}\right]\right\}^{\frac{1}{2}}+\left(b_{2}^{2}-\frac{1}{4}-c d\right)\right)^{-1} .
\end{aligned}
$$

Because $d<0$, Eq. (5.13) displays the feature that the minimum value $G_{m}$ and the determinant $\mathcal{D}^{\mathrm{PT}}$ have the same sign. The proof is complete.

\section{B. Separability}

We exploit the above-mentioned condition of separability: when a given TMGS is separable, then the function (5.7) is non-negative:

$$
G\left(\xi, \eta, u_{1}\right) \geqq 0 .
$$

If $d<0$, the minimum (5.13) is therefore non-negative,

$$
G_{m} \geqq 0,
$$

so that Simon's condition of separability (3.23) is fulfilled. As shown in Sec. IV, any state with $d \geqq 0$ observes the separability condition (4.48). Put together, this means that the EPR-like necessary condition of separability (5.14) implies the Peres-Simon inequality (3.24). We have reached this conclusion without any use of the PPT idea.

Conversely, according to Simon's separability criterion (3.24) any TMGS with $d \geqq 0$ is separable. By virtue of Eq. (5.13), it also implies that a TMGS with $d<0$ is separable if the inequality (5.15) is satisfied. As this condition is already proven to be necessary, it follows that the minimum $G_{m}$ is a separability indicator in its own right:

$$
G_{m} \geqq 0 \Longleftrightarrow \hat{\rho} \text { separable. }
$$

Equation (5.16) has the obvious alternative form:

$$
G_{m}<0 \Longleftrightarrow \hat{\rho} \text { entangled. }
$$

Finally, on account of Theorem 3, Simon's separability criterion for a TMGS, Eq. (4.48), entails that the identity (5.14) is not only a necessary, but also a sufficient condition of separability. 


\section{THE EPR-LIKE APPROACH TO SEPARABILITY REVISITED}

At this point it is useful to re-examine the full EPRlike approach to the separability of TMGSs put forward by Duan et al. in Ref. [14].

\section{A. Basic equations}

We start from the variances of the non-local oneparameter observables (1.5) given by Eqs. (4.21) and (4.22). Let us introduce the non-local operator

$$
\hat{P}(\alpha):= \begin{cases}\hat{P}_{+}(\alpha), & (d<0), \\ \hat{P}_{-}(\alpha), & (d \geqq 0) .\end{cases}
$$

Its variance,

$$
[\Delta P(\alpha)]^{2}=\frac{b_{1}}{u_{1}} \alpha^{2}+\frac{b_{2}}{u_{2}} \frac{1}{\alpha^{2}}-\frac{2|d|}{\sqrt{u_{1} u_{2}}},
$$

is the minimum over the pair of variances (4.22) with respect to the sign of $d$ :

$$
\Delta P(\alpha)=\min _{\{\operatorname{sgn}(d)\}}\left\{\Delta P_{+}(\alpha), \Delta P_{-}(\alpha)\right\} .
$$

Recall the definition of the signum function of a real variable:

$$
\operatorname{sgn}(x):=\left\{\begin{aligned}
-1, & (x<0) \\
0, & (x=0) \\
1, & (x>0)
\end{aligned}\right.
$$

Guided by the necessary conditions of separability 2.11 derived by Duan et al. in Ref. [14], we employ the following EPR-like correlation function, which is a regularized sum depending on the local scalings $u_{1}, u_{2}$ :

$$
\begin{aligned}
& K\left(\alpha^{2}, u_{1}, u_{2}\right):=[\Delta Q(\alpha)]^{2}+[\Delta P(\alpha)]^{2} \\
& -\left(\alpha^{2}+\frac{1}{\alpha^{2}}\right) .
\end{aligned}
$$

Substitution of the variances (4.1) and (4.2) into Eq. (6.4) gives the explicit formula:

$$
\begin{aligned}
& K\left(\alpha^{2}, u_{1}, u_{2}\right)=\alpha^{2}\left[b_{1}\left(u_{1}+\frac{1}{u_{1}}\right)-1\right] \\
& +\frac{1}{\alpha^{2}}\left[b_{2}\left(u_{2}+\frac{1}{u_{2}}\right)-1\right] \\
& -2\left(c \sqrt{u_{1} u_{2}}+\frac{|d|}{\sqrt{u_{1} u_{2}}}\right) .
\end{aligned}
$$

We apply the same pattern as in Sec. V, starting from the necessary condition of separability (2.11) which reads:

$$
K\left(\alpha^{2}, u_{1}, u_{2}\right) \geqq 0 \text {. }
$$

Our approach is to minimize the function (6.5). This reaches its minimum with respect to the variable $\alpha^{2}$ for the value

$$
\tilde{\alpha}_{m}^{2}=\sqrt{\frac{b_{2}\left(u_{2}+\frac{1}{u_{2}}\right)-1}{b_{1}\left(u_{1}+\frac{1}{u_{1}}\right)-1}} .
$$

The obtained minimum is a function of the scaling factors:

$$
\begin{aligned}
& f\left(u_{1}, u_{2}\right):=K\left(\tilde{\alpha}_{m}^{2}, u_{1}, u_{2}\right) \\
& =2\left\{\left[b_{1}\left(u_{1}+\frac{1}{u_{1}}\right)-1\right]\left[b_{2}\left(u_{2}+\frac{1}{u_{2}}\right)-1\right]\right\}^{\frac{1}{2}} \\
& -2\left(c \sqrt{u_{1} u_{2}}+\frac{|d|}{\sqrt{u_{1} u_{2}}}\right) .
\end{aligned}
$$

The stationarity conditions for the function (6.8) reduce to the following system of equations in the unknowns $u_{1}$ and $u_{2}$ :

$$
\frac{\frac{b_{1}}{u_{1}}-\frac{1}{2}}{b_{1} u_{1}-\frac{1}{2}}=\frac{\frac{b_{2}}{u_{2}}-\frac{1}{2}}{b_{2} u_{2}-\frac{1}{2}}
$$

$$
\begin{aligned}
& \sqrt{\left(b_{1} u_{1}-\frac{1}{2}\right)\left(b_{2} u_{2}-\frac{1}{2}\right)}-\sqrt{\left(\frac{b_{1}}{u_{1}}-\frac{1}{2}\right)\left(\frac{b_{2}}{u_{2}}-\frac{1}{2}\right)} \\
& =c \sqrt{u_{1} u_{2}}-\frac{|d|}{\sqrt{u_{1} u_{2}}} .
\end{aligned}
$$

Making use of Eq. (6.9), it is convenient to replace Eq. (6.10) by a polynomial one:

$$
b_{1} b_{2}\left(u_{1}^{2}-1\right)\left(u_{2}^{2}-1\right)=\left(c u_{1} u_{2}-|d|\right)^{2} .
$$

Equations (6.9) and (6.10) coincide with those written by Duan et al. in Ref. 14 in order to define what they have called the standard form II of the CM of a TMGS. We prefer instead the name witness standard form of the CM.As it will be shown in Sec. VI C, this seems to be more appropriate. We also term the local squeezings $\left\{\tilde{u}_{1}, \tilde{u}_{2}\right\}$ as witness scaling factors.

However, when trying to solve analytically the system under discussion in the general case, one faces a non-trivial algebraic equation of degree eight. For the time being, one does not know an explicit solution $\left\{\tilde{u}_{1}, \tilde{u}_{2}\right\}$ of Eqs. 6.9 and 6.11), except for some special classes of TMGSs, such as the thermal states (TSs), the symmetric ones [36], the mode-mixed thermal states (MTSs), and the STSs 37, 38], as well as the states subject to the constraint $\mathcal{D}:=\operatorname{det}\left(\mathcal{V}+\frac{i}{2} J\right)=0$ [28]. We also remark that the scaling factors written in Refs. 17, 18] are valid only for TMGSs at the separability threshold, i. e., fulfilling the condition $\mathcal{D}^{\mathrm{PT}}=0$. 


\section{B. Existence of a witness standard form $\mathcal{V}\left(\tilde{u}_{1}, \tilde{u}_{2}\right)$ of the covariance matrix}

It is extremely helpful to state here a theorem that sharpens a result of Ref. [14] regarding the existence of a solution of the above system in the general case.

Theorem 4. For any TMGS there exists at least a solution $\left\{\tilde{u}_{1}, \tilde{u}_{2}\right\}$ of the algebraic system (6.9) and (6.11) in the classicality range of the local squeeze factors:

$$
\tilde{u}_{1} \in\left[1,2 b_{1}\right], \quad \tilde{u}_{2} \in\left[1,2 b_{2}\right] .
$$

Such a solution determines awitness standard form of the $\mathrm{CM}: \mathcal{V}\left(\tilde{u}_{1}, \tilde{u}_{2}\right)$.

Proof. Note that Eq. (6.9) displays the pairings:

$$
u_{1}=1 \Longleftrightarrow u_{2}=1, \quad u_{1}=2 b_{1} \Longleftrightarrow u_{2}=2 b_{2} \text {. }
$$

As a matter of fact, Eq. [6.9) is a quadratic one in each of the variables $u_{1}$ and $u_{2}$, leading to a bijective continuous function,

$$
u_{2}=h\left(u_{1}\right), \quad h:\left[1,2 b_{1}\right] \longrightarrow\left[1,2 b_{2}\right],
$$

which reads:

$$
\begin{aligned}
& h\left(u_{1}\right)=\frac{2 b_{2} u_{1}\left(2 b_{1} u_{1}-1\right)}{b_{1}\left(u_{1}^{2}-1\right)+\sqrt{\Delta_{1}}}, \\
& \Delta_{1}:=b_{1}^{2}\left(u_{1}^{2}-1\right)^{2}+4 b_{2}^{2} u_{1}\left(2 b_{1}-u_{1}\right)\left(2 b_{1} u_{1}-1\right) .
\end{aligned}
$$

One gets the inverse function

$$
u_{1}=h^{-1}\left(u_{2}\right), \quad h^{-1}:\left[1,2 b_{2}\right] \longrightarrow\left[1,2 b_{1}\right],
$$

by interchanging the mode indices 1 and 2 in Eq. (6.14). The bijective function $h\left(u_{1}\right)$, Eq. (6.14), is strictly and continuously increasing from the initial value $h(1)=1$ to the final one $h\left(2 b_{1}\right)=2 b_{2}$. Remark that for a symmetric TMGS it reduces to the identity function:

$$
u_{2}=u_{1}, \quad\left(b_{1}=b_{2}=: b\right) .
$$

Coming back to the general case, we introduce a function of two variables suggested by Eq. (6.11):

$$
\Phi\left(u_{1}, u_{2}\right):=b_{1} b_{2}\left(u_{1}^{2}-1\right)\left(u_{2}^{2}-1\right)-\left(c u_{1} u_{2}-|d|\right)^{2} .
$$

The existence of a solution of the algebraic system (6.9) and 6.11 is equivalent to that of a zero of the onevariable function

$$
\phi\left(u_{1}\right):=\Phi\left(u_{1}, h\left(u_{1}\right)\right), \quad\left(u_{1} \in\left[1,2 b_{1}\right]\right) .
$$

In order to check if such a zero exists indeed, we have to examine the sign of the values of the function (6.18) at the end points of its domain:

$$
\phi(1)=\Phi(1,1)=-(c-|d|)^{2}: \quad \phi(1) \leqq 0 ;
$$

$$
\begin{aligned}
& \phi\left(2 b_{1}\right)=\Phi\left(2 b_{1}, 2 b_{2}\right) \\
= & 16 b_{1} b_{2}\left[\mathcal{D}+d^{2}\left(b_{1} b_{2}-c^{2}-\frac{1}{4}\right)+\frac{1}{2} c(|d|+d)\right] \\
& +4 d^{2}\left(b_{1} b_{2}-\frac{1}{4}\right): \quad \phi\left(2 b_{1}\right) \geqq 0 .
\end{aligned}
$$

The inequality in Eq. 6.20 stems from conditions (3.14) - (3.16), which are equivalent to the RobertsonSchrödinger uncertainty relation, Eq. (3.4). Therefore, by virtue of continuity, the function (6.18) has at least a zero in the classicality interval $u_{1} \in\left[1,2 b_{1}\right]$ for any TMGS. We denote the corresponding solution of Eqs. (6.9) and 6.11) by $\left\{\tilde{u}_{1}, \tilde{u}_{2}=h\left(\tilde{u}_{1}\right)\right\}$, so that

$$
\begin{aligned}
& \phi\left(\tilde{u}_{1}\right)=\Phi\left(\tilde{u}_{1}, \tilde{u}_{2}\right)=0, \\
& \left(\tilde{u}_{1} \in\left[1,2 b_{1}\right], \tilde{u}_{2} \in\left[1,2 b_{2}\right]\right) .
\end{aligned}
$$

This concludes the proof.

\section{Separability indicator}

The witness standard form of the $\mathrm{CM}$ is important because the condition of classicality for the corresponding TMGS,

$$
\mathcal{V}\left(\tilde{u}_{1}, \tilde{u}_{2}\right)-\frac{1}{2} I_{4} \geqslant 0,
$$

is equivalent to the requirement

$$
\tilde{f}:=f\left(\tilde{u}_{1}, \tilde{u}_{2}\right) \geqq 0 .
$$

Indeed, taking account of Eqs. 6.9 and (6.11), we get the twin formulae:

$$
\begin{aligned}
& \tilde{f}=4\left[\sqrt{\left(b_{1} \tilde{u}_{1}-\frac{1}{2}\right)\left(b_{2} \tilde{u}_{2}-\frac{1}{2}\right)}-c \sqrt{\tilde{u}_{1} \tilde{u}_{2}}\right], \\
& \tilde{f}=4\left[\sqrt{\left(\frac{b_{1}}{\tilde{u}_{1}}-\frac{1}{2}\right)\left(\frac{b_{2}}{\tilde{u}_{2}}-\frac{1}{2}\right)}-\frac{|d|}{\sqrt{\tilde{u}_{1} \tilde{u}_{2}}}\right] .
\end{aligned}
$$

We find it suitable to introduce the parallel notations:

$$
\begin{aligned}
& \tilde{f}^{\prime}:=4\left[\sqrt{\left(b_{1} \tilde{u}_{1}-\frac{1}{2}\right)\left(b_{2} \tilde{u}_{2}-\frac{1}{2}\right)}+c \sqrt{\tilde{u}_{1} \tilde{u}_{2}}\right]>0, \\
& \tilde{f}^{\prime \prime}:=4\left[\sqrt{\left(\frac{b_{1}}{\tilde{u}_{1}}-\frac{1}{2}\right)\left(\frac{b_{2}}{\tilde{u}_{2}}-\frac{1}{2}\right)}+\frac{|d|}{\sqrt{\tilde{u}_{1} \tilde{u}_{2}}}\right] \geqq 0 .
\end{aligned}
$$

The matrix condition (6.22) reduces to four inequalities:

$$
\begin{aligned}
& b_{1} \tilde{u}_{1}-\frac{1}{2} \geqq 0, \\
& \left(b_{1} \tilde{u}_{1}-\frac{1}{2}\right)\left(\frac{b_{1}}{\tilde{u}_{1}}-\frac{1}{2}\right) \geqq 0, \\
& \left(\frac{b_{1}}{\tilde{u}_{1}}-\frac{1}{2}\right) 4^{-2}\left(\tilde{f} \tilde{f}^{\prime}\right) \geqq 0, \\
& \operatorname{det}\left[\mathcal{V}\left(\tilde{u}_{1}, \tilde{u}_{2}\right)-\frac{1}{2} I_{4}\right]=4^{-4}\left(\tilde{f} \tilde{f}^{\prime}\right)\left(\tilde{f} \tilde{f}^{\prime \prime}\right) \geqq 0 .
\end{aligned}
$$


Three of them are already satisfied, so that the only condition to be fulfilled remains $\tilde{f} \geqq 0$, Eq. (6.23). This classicality requirement is a sufficient condition for the separability of the given TMGS $\hat{\rho}$, whose CM is congruent with its witness standard form $\mathcal{V}\left(\tilde{u}_{1}, \tilde{u}_{2}\right)$ via a local symplectic transformation, Eq. (3.8). Moreover, by virtue of Eq. 6.6), the inequality (6.23) is also a necessary condition of separability. Therefore, a TMGS whose CM has the witness standard form is a unique state for which separability reduces to classicality. By examining the sign of the EPR-like correlation function $\tilde{f}:=f\left(\tilde{u}_{1}, \tilde{u}_{2}\right)$, one can check whether the witness-standard-form TMGS is classical or not, i.e., whether it possesses or not a wellbehaved Glauber-Sudarshan $P$ representation.

To sum up, our optimization method has exploited the EPR-like correlation function (6.4) leading to the separability indicator $\tilde{f}$, Eq. 6.24). It is worth stressing that this indicator differs in two respects from the previous ones which are specified by Eqs. (4.19), 4.43), and (5.13). First, it has been identified independently of Simon's PPT separability criterion. Second, by contrast to the three indicators quoted above, we have not found yet in generality an explicit formula for the existing stationary point. We also are not quite sure that this is unique or that it corresponds to a minimum.

\section{Explicit witness scaling factors for special classes of states}

It is instructive to present concisely the special classes of TMGSs whose witness scaling factors can be explicitly evaluated, as mentioned in Sec. VI A.

1. TMGSs with $c=|d|$ : TSs, MTSs, and STSs. The solution

$$
\tilde{u}_{1}=\tilde{u}_{2}=1
$$

of Eqs. (6.9) and (6.11) is specific to this class, which consists of TSs $(c=d=0)$, MTSs $(c=d>$ $0)$, and $\operatorname{STSs}(c=-d>0)$. We have proven the uniqueness of the solution (6.27). Note the minimum value 6.7]:

$$
\tilde{\alpha}_{m}^{2}=\sqrt{\frac{b_{2}-\frac{1}{2}}{b_{1}-\frac{1}{2}}}
$$

Equations (6.24) and 6.25) give the separability indicator

$$
\tilde{f}=4\left[\sqrt{\left(b_{1}-\frac{1}{2}\right)\left(b_{2}-\frac{1}{2}\right)}-c\right],
$$

and, respectively, the functions

$$
\tilde{f}^{\prime}=\tilde{f}^{\prime \prime}=4\left[\sqrt{\left(b_{1}-\frac{1}{2}\right)\left(b_{2}-\frac{1}{2}\right)}+c\right] .
$$

Equation (6.28) becomes insightful when written in terms of the symplectic eigenvalues $\kappa_{ \pm}$for an MTS and $\kappa_{ \pm}^{\mathrm{PT}}$ for an STS:

$\tilde{f}_{\mathrm{MT}}=\frac{4^{2}}{\tilde{f}^{\prime}}\left(\kappa_{-}-\frac{1}{2}\right)\left(\kappa_{+}-\frac{1}{2}\right) \geqq 0, \quad(d>0)$,

$\tilde{f}_{\mathrm{ST}}=\frac{4^{2}}{\tilde{f}^{\prime}}\left(\kappa_{-}^{\mathrm{PT}}-\frac{1}{2}\right)\left(\kappa_{+}^{\mathrm{PT}}-\frac{1}{2}\right), \quad(d<0)$.

2. Symmetric TMGSs: $b_{1}=b_{2}=: b$.

We get a unique solution,

$$
\tilde{u}_{1}=\tilde{u}_{2}=\sqrt{\frac{b-|d|}{b-c}}
$$

which is specific to symmetric TMGSs, provided that $c>|d|$. The minimum value (6.7) of the parameter $\alpha$ is $\tilde{\alpha}_{m}=1$. We have proven the property of the solution (6.32) of being a minimum point of the function (6.8) written with $b_{1}=b_{2}=: b$. The corresponding absolute minimum is the EPR-like separability indicator

$$
\tilde{f}=4\left[\sqrt{(b-c)(b-|d|)}-\frac{1}{2}\right] .
$$

According to Eqs. (3.21) and (A5),

$\kappa_{-}=\sqrt{(b-c)(b-d)}, \quad \kappa_{-}^{\mathrm{PT}}=\sqrt{(b-c)(b+d)}$

so that the following relations hold:

$$
\begin{array}{r}
\tilde{f}=4\left(\kappa_{-}-\frac{1}{2}\right) \geqq 0, \quad(d \geqq 0), \\
\tilde{f}=4\left(\kappa_{-}^{\mathrm{PT}}-\frac{1}{2}\right), \quad(d<0) .
\end{array}
$$

3. TMGSs at the boundary $\tilde{f}=0$.

It will be shown in the next section that the property

$$
\tilde{f}=0
$$

is specific to all TMGSs with $d \geqq 0$ that are at the physicality edge $(\mathcal{D}=0)$ and to those with $d<0$ that are at the separability threshold $\left(\mathcal{D}^{\mathrm{PT}}=0\right)$. For both limit situations, we find a unique solution of the algebraic system (6.9) and 6.11):

$$
\begin{aligned}
& \tilde{u}_{1}=2 \frac{c\left(b_{1} b_{2}-d^{2}\right)+\frac{1}{4}|d|}{b_{1}|d|+b_{2} c}, \\
& \tilde{u}_{2}=2 \frac{c\left(b_{1} b_{2}-d^{2}\right)+\frac{1}{4}|d|}{b_{1} c+b_{2}|d|} .
\end{aligned}
$$


Let us focus on the expressions (6.30), (6.31), and (6.35) - 6.36) of the EPR-like correlation function $\tilde{f}$ for MTSs, STSs, and symmetric TMGSs, respectively. These important special GSs illustrate both cases: $d \geqq 0$ and $d<0$. The above explicit formulae clearly display the equivalence between the EPR-like separability condition (6.23) and the PPT one, Eq. (4.48). However, such explicit expressions are not available for an arbitrary TMGS.

\section{CONDITIONS OF SEPARABILITY: EPR-LIKE VERSUS PPT}

In principle, the EPR-like separability criterion (6.23) is as important as Simon's condition of separability (4.48) that relies on partial transposition. Nevertheless, it suffers from the drawback that, in the general case, it cannot be handled analytically. This makes Simon's PPT criterion of separability (4.48) to prevail in practice. However, the next theorem explicitly connects the EPR-like inequality (6.23) to the PPT one (4.48).

Theorem 5. The EPR-like separability condition (6.23) and Simon's PPT one, Eq. 4.48), are fully and manifestly equivalent.

Proof. We introduce the following function, which is symmetric in the mode indices 1 and 2 :

$$
\begin{aligned}
& Z\left(u_{1}, u_{2}\right):=\frac{1}{2}\left\{\left[\left(b_{1} u_{1}-\frac{1}{2}\right)\left(b_{2} u_{2}-\frac{1}{2}\right)-c^{2} u_{1} u_{2}\right]\right. \\
& \times\left[\left(\frac{b_{1}}{u_{1}}+\frac{1}{2}\right)\left(\frac{b_{2}}{u_{2}}+\frac{1}{2}\right)-\frac{d^{2}}{u_{1} u_{2}}\right] \\
& +\left[\left(b_{1} u_{1}+\frac{1}{2}\right)\left(b_{2} u_{2}+\frac{1}{2}\right)-c^{2} u_{1} u_{2}\right] \\
& \left.\times\left[\left(\frac{b_{1}}{u_{1}}-\frac{1}{2}\right)\left(\frac{b_{2}}{u_{2}}-\frac{1}{2}\right)-\frac{d^{2}}{u_{1} u_{2}}\right]\right\}
\end{aligned}
$$

The expression on the r. h. s. of Eq. (7.1) can be cast into a simpler form:

$$
Z\left(u_{1}, u_{2}\right)=H(d) \mathcal{D}+H(-d) \mathcal{D}^{\mathrm{PT}}+\frac{1}{4 u_{1} u_{2}} \Phi\left(u_{1}, u_{2}\right),
$$

where $H(x):=\frac{1}{2}[1+\operatorname{sgn}(x)]$ denotes the Heaviside step function and $\Phi\left(u_{1}, u_{2}\right)$ is the function (6.17). In view of Eqs. (3.16) and (3.23), we write the formula

$$
\begin{aligned}
H(d) \mathcal{D}+H(-d) \mathcal{D}^{\mathrm{PT}} & =\left(b_{1} b_{2}-c^{2}\right)\left(b_{1} b_{2}-d^{2}\right) \\
& -\frac{1}{4}\left(b_{1}^{2}+b_{2}^{2}+2 c|d|\right)+\frac{1}{16} .
\end{aligned}
$$

Let us equate the expressions (7.1) and (7.2) of the value $Z\left(\tilde{u}_{1}, \tilde{u}_{2}\right)$ corresponding to a witness standard form $\mathcal{V}\left(\tilde{u}_{1}, \tilde{u}_{2}\right)$ of the CM. Taking account of Eqs. (6.24),
(6.25), and 6.21), we get the identity:

$$
\begin{aligned}
& H(d) \mathcal{D}+H(-d) \mathcal{D}^{\mathrm{PT}} \\
& =\frac{1}{32} \tilde{f}\left\{\tilde{f}^{\prime}\left[\left(\frac{b_{1}}{\tilde{u}_{1}}+\frac{1}{2}\right)\left(\frac{b_{2}}{\tilde{u}_{2}}+\frac{1}{2}\right)-\frac{d^{2}}{\tilde{u}_{1} \tilde{u}_{2}}\right]\right. \\
& \left.+\tilde{f}^{\prime \prime}\left[\left(b_{1} \tilde{u}_{1}+\frac{1}{2}\right)\left(b_{2} \tilde{u}_{2}+\frac{1}{2}\right)-c^{2} \tilde{u}_{1} \tilde{u}_{2}\right]\right\} .
\end{aligned}
$$

In Eq. (7.4), the expression in curly brackets is strictly positive and $\mathcal{D} \geqq 0$ for any TMGS $\hat{\rho}$. Accordingly, the separability indicator $\tilde{f}$ and the local symplectic invariant (7.3) do have the same sign. There are two cases:

- $d \geqq 0$. Then $\tilde{f} \geqq 0$ and $\mathcal{D}^{\mathrm{PT}} \geqq 0$ : $\hat{\rho}$ separable;

- $d<0$.

Either $\tilde{f} \geqq 0 \Longleftrightarrow \mathcal{D}^{\mathrm{PT}} \geqq 0: \quad \hat{\rho}$ separable, or $\tilde{f}<0 \Longleftrightarrow \mathcal{D}^{\mathrm{PT}}<0$ : $\quad \hat{\rho}$ entangled.

As the signs of the separability indicators $\tilde{f}$ and $\mathcal{D}^{\text {PT }}$ coincide, we conclude they are equivalent in detecting separability of TMGSs:

$$
\begin{aligned}
& \tilde{f} \geqq 0 \quad \Longleftrightarrow \mathcal{D}^{\mathrm{PT}} \geqq 0: \quad \hat{\rho} \quad \text { separable; } \\
& \tilde{f}<0 \Longleftrightarrow \mathcal{D}^{\mathrm{PT}}<0: \quad \hat{\rho} \text { entangled. }
\end{aligned}
$$

The proof is complete. Note the equivalence

$$
\tilde{f}=0 \Longleftrightarrow H(d) \mathcal{D}+H(-d) \mathcal{D}^{\mathrm{PT}}=0,
$$

showing that the boundary $\tilde{f}=0$ consists of all TMGSs with $d \geqq 0$ that are at the physicality edge $(\mathcal{D}=0)$ as well as of all those with $d<0$ that are at the separability threshold $\left(\mathcal{D}^{\mathrm{PT}}=0\right)$.

Theorem 5 enforces the idea that the two approaches to the separability problem for TMGSs analyzed in this paper are fully equivalent. In spite of some recent assertions [17, 18] regarding the weakness of the EPR-like treatment in comparison to the PPT one, we have succeeded to prove their equivalence even in the absence of an explicit general solution for the witness scaling factors $\left\{\tilde{u}_{1}, \tilde{u}_{2}\right\}$. We also emphasize that Theorem 5 enables us to derive alternatively Simon's condition of separability for TMGSs without making any reference to the PPT formalism. Anyway, in order to decide whether a given TMGS is separable or not, then, by virtue of Eq. (7.5), one is entitled to employ Simon's condition of separability, $\mathcal{D}^{\mathrm{PT}} \geqq 0$, instead of the less efficient formula $\tilde{f} \geqq 0$.

\section{SUMMARY AND CONCLUSIONS}

This work is devoted to an explicit application of EPR-like correlations in detecting Gaussian entanglement. First, we tackle three correlation functions built with variances of two EPR-like observables in a TMGS. 
They are a normalized product, a normalized sum, and a non-normalized, but regularized sum. The corresponding analytic results are Theorems 1, 2, and 3, which express their absolute minima in terms of either the smallest symplectic eigenvalue $\kappa_{-}^{\mathrm{PT}}$ or the Simon determinant $\mathcal{D}^{\mathrm{PT}}$. These lower bounds are explicitly written in Eqs. (4.19), (4.43), and (5.13). On the one hand, we exploit them to point out that each of the three distinct EPR-like necessary conditions of separability for a TMGS implies the Peres-Simon PPT condition, Eq. 4.48). However, the EPR-like conditions are weaker than the latter because they do not imply its sufficiency. On the other hand, all three EPR-like correlations are separability indicators on account of Simon's PPT criterion of separability.

Due to their explicit expressions, Eqs. (4.19) and (4.43), both minimal normalized EPR-like uncertainties, besides being separability markers, give a definite physical significance to the smallest symplectic eigenvalue $\kappa_{-}^{\mathrm{PT}}$ of the CM of the partially transposed density matrix $\hat{\rho}^{\mathrm{PT}}$. Indeed, $\kappa_{-}^{\mathrm{PT}}$ appears to be a quantifier of the greatest amount of EPR-like correlations that can be created in any TMGS by means of local operations.

Second, we analyze the EPR-like approach of Duan et al. 14 by applying an optimization method which is similar to those employed in Secs. IV and V. This leads us to a new separability indicator for TMGSs. We then prove straightforwardly that the corresponding necessary and sufficient EPR-like condition of separability of a TMGS is fully equivalent to Simon's PPT one [3].

The EPR-like correlation function introduced in Ref. [14] is used in Sec. VI in a regularized sum form. An important conclusion is that its optimal value over the variables $\alpha, u_{1}, u_{2}$, denoted $\tilde{f}$, turns out to be a separability indicator for TMGSs. Recall that, among the original ideas put forward in Ref. [14], the central one is the existence of a witness standard form of the CM, which is confirmed here via Theorem 4 . The requirement of classicality for the corresponding privileged TMGS is equivalent to the separability of the whole set of TMGSs connected to it by local unitary transformations. Thus, the separability properties of the whole class of TMGSs having a given set of standard-form parameters are assigned to this witness-standard-form state. Our EPR-like separability indicator $\tilde{f}$, Eq. (6.24), is therefore obtained as a marker of classicality for the witness-standard-form TMGS. The resulting separability criterion (6.23) is quite special: indeed, it is independent of the PPT condition, by contrast to the preceding three separability criteria (4.49) and (5.16). However, in spite of its soundness, the original EPR-like approach [14] cannot decide analytically whether a TMGS is separable or not, except for the special cases discussed in Subsec. VI D. This happens because it does not provide a general analytic solution.

Another main result is Theorem 5, which explicitly proves that the EPR-like indicator of separability $\tilde{f}$ is equivalent to Simon's PPT separability marker $\mathcal{D}^{\mathrm{PT}}$. We have found a formula, Eq. (7.4), which reveals a direct connection between two distinct approaches to the sep- arability problem for TMGSs, providing valuable insight onto it. At the same time, it constitutes an alternative proof of Simon's PPT separability condition via the EPRlike correlation-function method. This new perspective might stimulate further research concerning the central role of the uncertainty relations in quantum mechanics. Needless to say, the importance of the EPR-like approach is enhanced by our simple proof of its manifest consistency with Simon's PPT separability condition, whose practical usefulness is universally acknowledged.

\section{Appendix A: Symplectic eigenvalues of the covariance matrix $\mathcal{V}^{\mathrm{PT}}$}

We focus on the positive definite $\mathrm{CM} \mathcal{V}^{\mathrm{PT}}$ which is built with the Gaussian operator $\hat{\rho}^{\mathrm{PT}}$ obtained from the TMGS $\hat{\rho}$ by partial transposition of the density matrix. Let us denote its symplectic eigenvalues by $\kappa_{ \pm}^{\mathrm{PT}}$ and write down the counterparts of Eqs. (3.18) and (3.19):

$$
\begin{gathered}
\operatorname{det}(\mathcal{V})=\left(\kappa_{+}^{\mathrm{PT}}\right)^{2}\left(\kappa_{-}^{\mathrm{PT}}\right)^{2}, \\
\mathcal{D}^{\mathrm{PT}}=\left[\left(\kappa_{+}^{\mathrm{PT}}\right)^{2}-\frac{1}{4}\right]\left[\left(\kappa_{-}^{\mathrm{PT}}\right)^{2}-\frac{1}{4}\right],
\end{gathered}
$$

with

$$
\kappa_{+}^{\mathrm{PT}} \geqq \kappa_{-}^{\mathrm{PT}}>0
$$

from Eqs. A1 , A2 , and (3.24) we get the biquadratic equation

$$
\left(\kappa^{\mathrm{PT}}\right)^{4}-\left(b_{1}^{2}+b_{2}^{2}-2 c d\right)\left(\kappa^{\mathrm{PT}}\right)^{2}+\operatorname{det}(\mathcal{V})=0,
$$

satisfied by the symplectic eigenvalues:

$$
\begin{aligned}
& \left(\kappa_{ \pm}^{\mathrm{PT}}\right)^{2}=\frac{1}{2}\left[\left(b_{1}^{2}+b_{2}^{2}-2 c d\right) \pm \sqrt{\Delta^{\mathrm{PT}}}\right], \\
& \Delta^{\mathrm{PT}}:=\left(b_{1}^{2}+b_{2}^{2}-2 c d\right)^{2}-4 \operatorname{det}(\mathcal{V}) \\
& =\left(b_{1}^{2}-b_{2}^{2}\right)^{2}+4\left(b_{1} c-b_{2} d\right)\left(b_{2} c-b_{1} d\right) \geqq 0 .
\end{aligned}
$$

Taking account of Eqs. A2 and A3 Simon's separability condition (3.24) states the following alternative:

- $\mathcal{D}^{\mathrm{PT}} \geqq 0 \quad \Longleftrightarrow \quad \kappa_{-}^{\mathrm{PT}} \geqq \frac{1}{2} \quad$ for separable TMGSs;

- $\mathcal{D}^{\mathrm{PT}}<0 \Longleftrightarrow \kappa_{-}^{\mathrm{PT}}<\frac{1}{2} \quad$ for entangled TMGSs.

Note that the partial transpose $\hat{\rho}^{\mathrm{PT}}$ of any entangled TMGS $\hat{\rho}$ is no longer a state.

Multiplication of Eq. (A4) by the positive quantities $b_{1} b_{2}-c^{2}$ and $b_{1} b_{2}-d^{2}$ from Eq. (3.15) yields two useful identities:

$$
\begin{aligned}
& {\left[b_{1}\left(b_{1} b_{2}-c^{2}\right)-b_{2}\left(\kappa_{ \pm}^{\mathrm{PT}}\right)^{2}\right]\left[b_{2}\left(b_{1} b_{2}-c^{2}\right)-b_{1}\left(\kappa_{ \pm}^{\mathrm{PT}}\right)^{2}\right]} \\
& =\left[c\left(\kappa_{ \pm}^{\mathrm{PT}}\right)^{2}-d\left(b_{1} b_{2}-c^{2}\right)\right]^{2} ;
\end{aligned}
$$


$\left[b_{1}\left(b_{1} b_{2}-d^{2}\right)-b_{2}\left(\kappa_{ \pm}^{\mathrm{PT}}\right)^{2}\right]\left[b_{2}\left(b_{1} b_{2}-d^{2}\right)-b_{1}\left(\kappa_{ \pm}^{\mathrm{PT}}\right)^{2}\right]$
$=\left[c\left(b_{1} b_{2}-d^{2}\right)-d\left(\kappa_{ \pm}^{\mathrm{PT}}\right)^{2}\right]^{2}$.

Equations (A6) and (A7) transform into each other by interchanging the parameters $c$ and $-d$. Another pair of identities related in the same way is obtained when we multiply Eq. (A4) by the product $b_{1} b_{2}$ :

$$
\begin{aligned}
& {\left[b_{1}\left(b_{1} b_{2}-c^{2}\right)-b_{2}\left(\kappa_{ \pm}^{\mathrm{PT}}\right)^{2}\right]\left[b_{2}\left(b_{1} b_{2}-d^{2}\right)-b_{1}\left(\kappa_{ \pm}^{\mathrm{PT}}\right)^{2}\right]} \\
& =\left(b_{1} c-b_{2} d\right)^{2}\left(\kappa_{ \pm}^{\mathrm{PT}}\right)^{2} ; \\
& {\left[b_{1}\left(b_{1} b_{2}-d^{2}\right)-b_{2}\left(\kappa_{ \pm}^{\mathrm{PT}}\right)^{2}\right]\left[b_{2}\left(b_{1} b_{2}-c^{2}\right)-b_{1}\left(\kappa_{ \pm}^{\mathrm{PT}}\right)^{2}\right]} \\
& =\left(b_{2} c-b_{1} d\right)^{2}\left(\kappa_{ \pm}^{\mathrm{PT}}\right)^{2} .
\end{aligned}
$$

We finally mention an identity involving the determinant (A2):

$$
\begin{aligned}
& 4\left[b_{2}\left(b_{1} b_{2}-c^{2}\right)-\frac{1}{4} b_{1}\right]\left[b_{2}\left(b_{1} b_{2}-d^{2}\right)-\frac{1}{4} b_{1}\right] \\
& -\left(b_{2}^{2}-\frac{1}{4}-c d\right)^{2}=4\left(b_{2}^{2}-\frac{1}{4}\right) \mathcal{D}^{\mathrm{PT}} .
\end{aligned}
$$

\section{Appendix B: Hessian matrices}

\section{The function $\ln [E(\lambda, \mu)]$}

By using Eq. (4.11), we evaluate the second-order derivatives of the function $\ln [E(\lambda, \mu)]$ at the stationary point (4.17):

$$
\begin{aligned}
& H_{11}:=\frac{\partial^{2} \ln (E)}{\partial \lambda^{2}}\left(\lambda_{m}, \mu_{m}\right), H_{22}:=\frac{\partial^{2} \ln (E)}{\partial \mu^{2}}\left(\lambda_{m}, \mu_{m}\right), \\
& H_{12}:=\frac{\partial^{2} \ln (E)}{\partial \lambda \partial \mu}\left(\lambda_{m}, \mu_{m}\right) .
\end{aligned}
$$

By use of Eq. (4.16), we get the following entries of the Hessian matrix:

$$
\begin{aligned}
& H_{11}=\frac{2\left(b_{1} b_{2}-c^{2}\right)}{\left[\Delta Q\left(\lambda_{m}\right)\right]^{4}}>0, \quad H_{22}=\frac{2\left(b_{1} b_{2}-d^{2}\right)}{\left[\Delta P\left(\mu_{m}\right)\right]^{4}}>0, \\
& H_{12}=-\frac{2}{\left(1+\lambda_{m} \mu_{m}\right)^{2}}<0 .
\end{aligned}
$$

Then, in view of Eq. A5), the Hessian determinant is positive:

$$
\operatorname{det}(H)=\frac{4 \sqrt{\Delta^{\mathrm{PT}}}}{\left(\kappa_{-}^{\mathrm{PT}}\right)^{2}\left(1+\lambda_{m} \mu_{m}\right)^{4}}>0 .
$$

Consequently, the Hessian matrix (B1) is positive definite. The absolute minimum of the function $E(\lambda, \mu)$, Eq. (4.11), is therefore

$$
E_{m}=E\left(\lambda_{m}, \mu_{m}\right) .
$$

\section{The function $F\left(\alpha^{2}, u_{1}, u_{2}\right)$}

We have evaluated the Hessian matrix of the function $F\left(\alpha^{2}, u_{1}, u_{2}\right)$, Eq. (4.23), at its stationary point, Eqs. (4.40) and (4.41). In the sequel, the indices $1,2,3$ refer to the independent variables $u_{1}, u_{2}, \alpha^{2}$, respectively. Let us write the expressions of three principal minors: the diagonal entry $H_{11}$, the cofactor $A_{33}$, and the Hessian determinant $\operatorname{det}(H)$. First, the following expression of $H_{11}$ holds provided that $c+d>0$ :

$$
\begin{aligned}
& H_{11}=\frac{\sqrt{\gamma}}{2\left(\alpha_{m}^{2}+\frac{1}{\alpha_{m}^{2}}\right) u_{1 m}^{2} u_{2 m}} \\
& \times\left[\left(1+\frac{1}{u_{1 m}^{2}}\right) \frac{c u_{1 m} u_{2 m}+d}{1-\frac{1}{u_{1 m}^{2}}}+2 \frac{c \gamma+d}{1-\frac{1}{u_{1 m}^{2}}}\right]>0 .
\end{aligned}
$$

A suitable simplification in Eq. (B5) yields a formula which is valid also in the limit case of the two-mode STSs $(c+d=0)$ :

$$
\begin{aligned}
& H_{11}=\frac{\sqrt{\gamma}}{2\left(\alpha_{m}^{2}+\frac{1}{\alpha_{m}^{2}}\right) u_{1 m}^{2} u_{2 m}} \\
& \times \frac{1}{b_{1} \sqrt{\Delta^{\mathrm{PT}}}+b_{1}\left(b_{1}^{2}-b_{2}^{2}\right)-2 d\left(b_{1} c-b_{2} d\right)} \\
& \times\left\{( 1 + \frac { 1 } { u _ { 1 m } ^ { 2 } } ) \frac { b _ { 1 } ( b _ { 1 } b _ { 2 } - d ^ { 2 } ) } { \gamma ( b _ { 1 } b _ { 2 } - c ^ { 2 } ) } \left[c\left(b_{1}^{2}+b_{2}^{2}\right)-d\left(2 b_{1} b_{2}\right)\right.\right. \\
& \left.\left.+c \sqrt{\Delta^{\mathrm{PT}}}\right]+4\left(b_{1} b_{2}-d^{2}\right)\left(b_{1} c-b_{2} d\right)\right\}>0 .
\end{aligned}
$$

Second, we present a general symmetric expression of the cofactor $A_{33}$ :

$$
\begin{aligned}
& A_{33}=\frac{1}{\left(\alpha_{m}^{2}+\frac{1}{\alpha_{m}^{2}}\right)^{2} u_{1 m} u_{2 m}}\left\{b_{1} b_{2}\left(\frac{1}{u_{2 m}}-\frac{1}{u_{1 m}}\right)^{2}\right. \\
& +\frac{4\left(b_{1} b_{2}-c^{2}\right)\left(b_{1} c-b_{2} d\right)\left(b_{2} c-b_{1} d\right)}{c\left(b_{1}^{2}+b_{2}^{2}\right)-d\left(2 b_{1} b_{2}\right)+c \sqrt{\Delta^{\mathrm{PT}}}} \\
& \left.\times \frac{1}{c-d}\left(1+\frac{1}{u_{1 m} u_{2 m}}\right)\right\}>0 .
\end{aligned}
$$

Third, we have obtained the Hessian determinant:

$$
\begin{aligned}
& \operatorname{det}(H)=\frac{4}{\alpha_{m}^{4}\left(\alpha_{m}^{2}+\frac{1}{\alpha_{m}^{2}}\right)^{3}\left(u_{1 m} u_{2 m}\right)^{\frac{3}{2}}} \\
& \times \frac{4\left(b_{1} b_{2}-c^{2}\right)\left(b_{1} c-b_{2} d\right)\left(b_{2} c-b_{1} d\right)}{c\left(b_{1}^{2}+b_{2}^{2}\right)-d\left(2 b_{1} b_{2}\right)+c \sqrt{\Delta^{\mathrm{PT}}}}>0 .
\end{aligned}
$$

The obvious inequalities (B6)- (B8) show that the Hessian matrix under discussion is positive definite. Therefore, the unique stationary point $\left\{\alpha_{m}^{2}, u_{1 m}, u_{2 m}\right\}$ is a minimum point where the function $F\left(\alpha^{2}, u_{1}, u_{2}\right)$ reaches its absolute minimum (4.42).

Needless to say, Eqs. (B6)- (B8) considerably simplify in the particular cases of STSs and symmetric states. We list the resulting formulae as follows. 
1. Two-mode STSs:

$$
\begin{aligned}
& H_{11}=\frac{1}{\sqrt{\delta}}\left\{b_{1} \sqrt{\delta}-\left[b_{1}\left(b_{1}-b_{2}\right)+c^{2}\right]\right\}>0 \\
& A_{33}=\frac{c^{2}}{\delta}\left(b_{1}+b_{2}\right)\left[\left(b_{1}+b_{2}\right)-\sqrt{\delta}\right]>0 ; \\
& \operatorname{det}(H)=\delta^{-\frac{3}{2}} c^{2}\left(b_{1}+b_{2}\right)\left[\sqrt{\delta}+\left(b_{1}-b_{2}\right)\right]^{2} \\
& \times\left[\left(b_{1}+b_{2}\right)-\sqrt{\delta}\right]>0 .
\end{aligned}
$$

\section{Symmetric TMGSs:}

$$
\begin{aligned}
& H_{11}=\frac{1}{4}\left(\frac{b-c}{b+d}\right)^{\frac{1}{2}} \frac{1}{b+d}\{b[(b-c)+(b+d)] \\
& +2(b-c)(b+d)\}>0 ; \\
& A_{33}=\frac{1}{2}\left(\frac{b-c}{b+d}\right)^{2} b[(b-c)+(b+d)]>0 ; \\
& \operatorname{det}(H)=\left(\frac{b-c}{b+d}\right)^{\frac{3}{2}} b(b-c)(c-d)>0 .
\end{aligned}
$$

The above formulae have been checked by direct evaluation of the corresponding Hessian matrices starting from Eqs. (4.44) and (4.45), respectively.

\section{The function $G\left(\xi, \eta, u_{1}\right)$}

Let us assign the indices 1, 2, 3 to the independent variables $u_{1}, \xi, \eta$, respectively. The Hessian matrix of the function $G\left(\xi, \eta, u_{1}\right)$, Eq. (5.7), evaluated at the stationary point (5.10) has the following entries:

$$
\begin{aligned}
& H_{11}=\frac{1}{\left(b_{2}^{2}-\frac{1}{4}\right) u_{1 m}^{3}}\left\{\left[b_{2}\left(b_{1} b_{2}-d^{2}\right)-\frac{1}{4} b_{1}\right]\right. \\
& +\frac{1}{2}\left[b_{2}\left(b_{1} b_{2}-c^{2}\right)-\frac{1}{4} b_{1}\right]\left(u_{1 m}^{2}+1\right) \\
& \left.+\frac{1}{2} b_{2} c^{2}\left(u_{1 m}-1\right)^{2}+\frac{1}{2} c\left(2 b_{2} c+d\right) u_{1 m}\right\}>0, \\
& H_{22}=2 b_{2}, \quad H_{33}=2 b_{2}, \\
& H_{12}=-c u_{1 m}^{-\frac{1}{2}}, \quad H_{13}=-d u_{1 m}^{-\frac{3}{2}}, \quad H_{23}=-1 .
\end{aligned}
$$

Three nested principal minors are clearly positive: the diagonal entry $H_{33}$, the cofactor $A_{11}$, and the Hessian determinant $\operatorname{det}(H)$. Indeed,

$$
\begin{aligned}
& H_{33}=2 b_{2}>0, \quad A_{11}=4\left(b_{2}^{2}-\frac{1}{4}\right)>0, \\
& \operatorname{det}(H)=\frac{2}{u_{1 m}^{3}}\left\{2\left[b_{2}\left(b_{1} b_{2}-d^{2}\right)-\frac{1}{4} b_{1}\right]\right. \\
& +\left[b_{2}\left(b_{1} b_{2}-c^{2}\right)-\frac{1}{4} b_{1}\right]\left(u_{1 m}^{2}+1\right) \\
& \left.+b_{2}\left(c^{2}-d^{2}\right)\right\}>0 .
\end{aligned}
$$

Accordingly, the Hessian matrix with the entries (B12) is positive definite. This means that the unique stationary point $\left\{\xi_{m}, \eta_{m}, u_{1 m}\right\}$, Eq. (5.10), is a minimum point where the function $G\left(\xi, \eta, u_{1}\right)$ reaches its absolute minimum (5.11).

Acknowledgments: This work was supported by the funding agency CNCS-UEFISCDI of the Romanian Ministry of Research and Innovation through Grant No. PNIII-P4-ID-PCE-2016-0794.
[1] Peres A 1996 Separability Criterion for Density Matrices Phys. Rev. Lett. 771413

[2] Horodecki M, Horodecki P, Horodecki R 1996 Separability of mixed states: necessary and sufficient conditions Phys. Lett. A 2231

[3] Simon R 2000 Peres-Horodecki Separability Criterion for Continuous Variable Systems Phys. Rev. Lett. 842726

[4] Shchukin E and Vogel W 2005 Inseparability Criteria for Continuous Bipartite Quantum States Phys. Rev. Lett. 95 230502; Erratum 2006 Phys. Rev. Lett. 96129902

[5] Agarwal G S and Biswas A 2005 Inseparability inequalities for higher order moments for bipartite systems New J.Phys. 7211

[6] Hillery M and Zubairy M S 2006 Entanglement Conditions for Two-Mode States Phys. Rev. Lett. 96050503

[7] Einstein A, Podolsky B and Rosen N 1935 Can QuantumMechanical Description of Physical Reality Be Considered Complete? Phys. Rev. 47777

[8] Reid M D 1989 Demonstration of the Einstein-PodolskyRosen paradox using nondegenerate parametric amplification Phys. Rev. A 40913
[9] Reid M D, Drummond P D, Bowen W P, Cavalcanti E G, Lam P K, Bachor H A, Andersen U L and Leuchs G 2009 Colloquium: The Einstein-Podolsky-Rosen paradox: From concepts to applications Rev. Mod. Phys. 81 1727

[10] Schrödinger E 1935 Discussion of Probability Relations between Separated Systems (I) Math. Proc. Camb. Phil. Soc. 31555

[11] Wiseman H M, Jones S J and Doherty A C 2007 Steering, Entanglement, Nonlocality, and the Einstein-PodolskyRosen Paradox Phys. Rev. Lett. 98140402

[12] Jones S J, Wiseman H M and Doherty A C 2007 Entanglement, Einstein-Podolsky-Rosen correlations, Bell nonlocality, and steering Phys. Rev. A 76052116

[13] Cavalcanti E G, Jones S J, Wiseman H M and Reid M D 2009 Experimental criteria for steering and the EinsteinPodolsky-Rosen paradox Phys. Rev. A 80032112

[14] Duan L-M, Giedke G, Cirac J I and Zoller P 2000 Inseparability Criterion for Continuous Variable Systems Phys. Rev. Lett. 842722 
[15] Mancini S, Giovannetti V, Vitali D and Tombesi P 2002 Entangling Macroscopic Oscillators Exploiting Radiation Pressure Phys. Rev. Lett. 88120401

[16] Giovannetti V, Mancini S, Vitali D and Tombesi P 2003 Characterizing the entanglement of bipartite quantum states Phys. Rev. A $\mathbf{6 7} 022320$

[17] Fujikawa K 2009 Separability criteria for continuousvariable systems Phys. Rev. A 80012315

[18] Fujikawa K 2012 Explicit formula of the separability criterion for continuous variables systems Int. J. Mod. Phys. B 261243006

[19] Bowen W P, Treps N, Schnabel R and Lam P K 2002 Experimental Demonstration of Continuous Variable Polarization Entanglement Phys. Rev. Lett. 89253601

[20] Josse V, Dantan A, Bramati A, Pinard M and Giacobino E 2004 Continuous Variable Entanglement using Cold Atoms Phys. Rev. Lett. 92123601

[21] Buono D, Nocerino G, Porzio A and Solimeno S 2012 Experimental analysis of decoherence in continuous-variable bipartite systems Phys. Rev. A 86042308

[22] Eisert J and Plenio M B 2003 Introduction to the basics of entanglement theory in continuous-variable systems Int. J. Quantum Inform. 01479

[23] Braunstein S L and van Loock P 2005 Quantum information with continuous variables Rev. Mod. Phys. 77513

[24] Adesso G, Ragy S and Lee A R 2014 Continuous Variable Quantum Information: Gaussian States and Beyond Open Syst. Inf. Dyn. 211440001

[25] Marian P, Marian T A and Scutaru H 2001 Inseparability of mixed two-mode Gaussian states generated with a SU $(1,1)$ interferometer J. Phys. A: Math. Gen. 346969

[26] Adesso G, Bromley T R and Cianciaruso M 2016 Measures and applications of quantum correlations J. Phys. A: Math. Theor. 49473001
[27] Marian P, Marian T A and Scutaru H 2003 Bures distance as a measure of entanglement for two-mode squeezed thermal states Phys. Rev. A 68062309

[28] Adesso G, Serafini A and Illuminati F 2004 Extremal entanglement and mixedness in continuous variable systems Phys. Rev. A $\mathbf{7 0} 022318$

[29] Williamson J 1936 On the Algebraic Problem Concerning the Normal Forms of Linear Dynamical Systems Amer. J. Math. 58141

[30] Vidal G and Werner R F 2002 Computable measure of entanglement Phys. Rev. A 65032314

[31] Poon P S Y and Law C K 2007 Negativity of asymmetric two-mode Gaussian states: An explicit analytic formula and physical interpretation Phys. Rev. A 76054305

[32] He Q Y and Reid M D 2013 Einstein-Podolsky-Rosen paradox and quantum steering in pulsed optomechanics Phys. Rev. A $\mathbf{8 8} 052121$

[33] Kiesewetter S, He Q Y, Drummond P D and Reid M D 2014 Scalable quantum simulation of pulsed entanglement and Einstein-Podolsky-Rosen steering in optomechanics Phys. Rev. A $\mathbf{9 0} 043805$

[34] He Q Y, Gong Q H and Reid M D 2015 Classifying Directional Gaussian Entanglement, Einstein-Podolsky-Rosen Steering, and Discord Phys. Rev. Lett. 114060402

[35] Kogias I and Adesso G 2015 Einstein-Podolsky-Rosen steering measure for two-mode continuous variable states J. Opt. Soc. Am. B 32 A 27

[36] Marian P and Marian T A 2008 Bures distance as a measure of entanglement for symmetric two-mode Gaussian states Phys. Rev. A $7 \mathbf{7 0 6 2 3 1 9}$

[37] Marian P and Marian T A 2015 Hellinger distance as a measure of Gaussian discord J. Phys. A: Math. Theor. 48115301

[38] Marian P and Marian T A 2016 Quantum Fisher information on two manifolds of two-mode Gaussian states Phys. Rev. A 93052330 